\title{
Ideal clutters that do not pack
}

\author{
Ahmad Abdi \\ Gérard Cornuéjols \\ Kanstantsin Pashkovich
}

June 24, 2018

\begin{abstract}
For a clutter $\mathcal{C}$ over ground set $E$, a pair of distinct elements $e, f \in E$ are coexclusive if every minimal cover contains at most one of them. An identification of $\mathcal{C}$ is another clutter obtained after identifying coexclusive elements of $\mathcal{C}$. If a clutter is non-packing, then so is any identification of it.

Inspired by this observation, and impelled by the lack of a qualitative characterization for ideal minimally non-packing (mnp) clutters, we reduce ideal mnp clutters even further by taking their identifications. In doing so, we reveal chains of ideal mnp clutters, demonstrate the centrality of mnp clutters with covering number two, as well as provide a qualitative characterization of irreducible ideal mnp clutters with covering number two. At the core of this characterization lies a class of objects, called marginal cuboids, that naturally give rise to ideal non-packing clutters with covering number two. We present an explicit class of marginal cuboids, and show that the corresponding clutters have one of $Q_{6}, Q_{2,1}, Q_{10}$ as a minor, where $Q_{6}, Q_{2,1}$ are known ideal mnp clutters, and $Q_{10}$ is a new ideal mnp clutter.
\end{abstract}

\section{Introduction}

Let $E$ be a finite set of elements, and $\mathcal{C}$ a family of subsets of $E$, called members. We say $\mathcal{C}$ is a clutter over ground set $E=: E(\mathcal{C})$ if no member is contained in another one. The incidence matrix $M(\mathcal{C})$ of clutter $\mathcal{C}$ is a $0-1$ matrix whose columns are labeled by $E(\mathcal{C})$ and whose rows are the incidence vectors of the members. A cover is an subset of $E$ that intersects every member of $\mathcal{C}$. The blocker of $\mathcal{C}$, denoted $b(\mathcal{C})$, is another clutter over the same ground set whose members consist of the (inclusion-wise) minimal covers of $\mathcal{C}$. It is well-known that the blocker of $b(\mathcal{C})$ is $\mathcal{C}$ itself, i.e. $b(b(\mathcal{C}))=\mathcal{C}[7,10]$

The covering number, denoted $\tau(\mathcal{C})$, is the minimum size of a cover. A packing is a collection of pairwise disjoint members. The packing number, denoted $\nu(\mathcal{C})$, is the maximum size of a packing. For instance, the clutter $\{\{1,2\},\{2,3\},\{3,1\}\}$ has covering number 2 and packing number 1 . Since a cover picks an element from each member of a packing, we get that

$$
\tau(\mathcal{C}) \geq \nu(\mathcal{C})
$$

When equality holds above, we say that $\mathcal{C}$ packs. Broadly speaking, we would like to study the structure of clutters that do not pack, but as the reader may expect, more often than not clutters do not pack, so we cannot 
hope to find structure in such clutters. We will therefore adjust our expectations and settle for a substructure analysis of such clutters. Let us explain what substructure means.

Fix an element $e$. The deletion $\mathcal{C} \backslash e$ is the clutter over ground set $E-\{e\}$ whose members are $\{C: e \notin$ $C \in \mathcal{C}\}$. The contraction $\mathcal{C} / e$ is the clutter over ground set $E-\{e\}$ whose members are the minimal sets in $\{C-\{e\}: C \in \mathcal{C}\}$. It can be readily checked that $b(\mathcal{C} \backslash e)=b(\mathcal{C}) / e$ and $b(\mathcal{C} / e)=b(\mathcal{C}) \backslash e$ [18]. Fix disjoint $I, J \subseteq E$. The clutter $\mathcal{C} \backslash I / J$ obtained after deleting elements $I$ and contracting elements $J$ is called a minor of $\mathcal{C}$ (changing the order of deletions and contractions does not change the minor). When $I \cup J \neq \emptyset, \mathcal{C} \backslash I / J$ is called a proper minor. Moreover, for convenience, we call $\mathcal{C}$ a major of $\mathcal{C} \backslash I / J$.

A clutter is minimally non-packing (mnp) if it does not pack but every proper minor of it does. Notice that every clutter that does not pack has an mnp minor. The reader may now hope that mnp clutters possess identifiable structure, and to a certain extent this is correct. A seminal result of Lehman [12] implies that,

Theorem 1.1 (see [5]). A minimally non-packing clutter is either ideal or minimally non-ideal.

Clutter $\mathcal{C}$ is ideal if the polyhedron

$$
\begin{aligned}
Q(\mathcal{C}): & =\left\{x \in \mathbb{R}_{+}^{E}: x(C) \geq 1 \forall C \in \mathcal{C}\right\} \\
& =\left\{x \in \mathbb{R}_{+}^{E}: M(\mathcal{C}) x \geq \mathbf{1}\right\}
\end{aligned}
$$

has only integral extreme points; otherwise it is non-ideal. (Here, $x(C)=\sum_{e \in C} x_{e}$.) Lehman's Width-Length Inequality [11] (also see Fulkerson [8]) implies that if $\mathcal{C}$ is ideal, then so is $b(\mathcal{C})$. We say a clutter is minimally non-ideal (mni) if it is non-ideal but every proper minor of it is ideal. Lehman [12] (also see Seymour [17]) showed that these clutters possess a lot of structure, and his structure is qualitative in the sense that it explains why such clutters are non-ideal and do not pack. ${ }^{1}$ Therefore, in light of the result above, we consider non-ideal mnp clutters well-understood, and focus on ideal mnp clutters.

Besides a dozen such clutters, Cornuéjols, Guenin and Margot [5] found an infinite class of ideal mnp clutters $\left\{Q_{r, t}: r, t \in \mathbb{N}\right\}$ that will be defined explicitly in $\S 5.2$ - all these clutters have covering number two. (They in fact conjecture that all ideal mnp clutters have this property.) Other than this intriguing common feature, a qualitative structure result for these clutters remains elusive. An explanation for this shortfall is that being ideal is a global property and difficult to take advantage of. We will come up with a compromise - let us elaborate.

Being ideal is also a minor-closed property [19]. By using the fact that ideal clutters exclude certain nonideal minors, we will extract some local structure in ideal mnp clutters. This local structure will in turn allow us to reduce ideal mnp clutters even further to the extent where a qualitative characterization is achieved.

\subsection{Deltas, coexclusive elements and identifications}

Take an integer $n \geq 3$. A delta of dimension $n$, denoted $\Delta_{n}$, is the clutter over ground set $[n]:=\{1,2,3, \ldots, n\}$ whose members are

$$
\Delta_{n}=\{\{1,2\},\{1,3\}, \cdots,\{1, n\},\{2,3, \ldots, n\}\},
$$

\footnotetext{
${ }^{1}$ In fact, Cornuéjols et al. [5] proved that a non-ideal mnp clutter is either a delta or an mni clutter whose core is thin.
} 
with incidence matrix

$$
M\left(\Delta_{n}\right)=\left(\begin{array}{ccccc}
1 & 1 & & & \\
1 & & 1 & & \\
\vdots & & & \ddots & \\
1 & & & & 1 \\
& 1 & 1 & \ldots & 1
\end{array}\right) .
$$

The deltas are an important class of (minimally) non-ideal clutters, ${ }^{2}$ and we believe that understanding when clutters do (not) have delta minors is crucial to the theory of clutters. In $\S 2$, we provide a tool for finding a delta minor, and show how this tool gives rise to a polynomial time algorithm for detecting delta minors. We will also see how excluding delta minors leads to local structure.

We say distinct elements $e, f$ are exclusive in $\mathcal{C}$ if every member contains at most one of $e, f$, and that they are coexclusive in $\mathcal{C}$ if every minimal cover contains at most one of $e, f$. Notice that $e, f$ are coexclusive in $\mathcal{C}$ if and only if they are exclusive in $b(\mathcal{C})$. The following theorem summarizes all that we need to know about coexclusive elements and how they enforce local structure:

Theorem 1.2. Let $\mathcal{C}$ be a clutter and take distinct elements $e, f$. The following statements are equivalent:

(i) e, f are coexclusive,

(ii) for all members $C_{e}, C_{f}$ such that $C_{e} \cap\{e, f\}=\{e\}$ and $C_{f} \cap\{e, f\}=\{f\},\left(C_{e} \cup C_{f}\right)-\{e, f\}$ contains another member,

(iii) for every extreme point $x^{\star}$ of $Q(\mathcal{C}), x_{e}^{\star}+x_{f}^{\star} \leq 1$.

This theorem is proved in $\S 3$. As an immediate consequence of Theorem 1.2 (ii),

Remark 1.3. Let $\mathcal{C}$ be a clutter over ground set $E$. Then in time $O\left(|E|^{2}|\mathcal{C}|^{3}\right)$, one can find a pair of coexclusive elements or certify that none exists.

Take coexclusive elements $e, f$ of $\mathcal{C}$. The identification $\left.\mathcal{C}\right|_{e=f}$ is the clutter over ground set $E-\{f\}$ whose members are the minimal sets in

$$
\{C: f \notin C \in \mathcal{C}\} \cup\{(C \cup\{e\})-\{f\}: f \in C \in \mathcal{C}\}
$$

Remark 1.4. Let $\mathcal{C}$ be a clutter with coexclusive elements $e, f$. The following statements hold:

(i) Let $B$ be a cover of $\mathcal{C}$. If $f \notin B$, then $B$ is a cover of $\left.\mathcal{C}\right|_{e=f}$, and if $f \in B$, then $(B \cup\{e\})-\{f\}$ is a cover of $\left.\mathcal{C}\right|_{e=f}$.

(ii) Let $K$ be a cover of $\left.\mathcal{C}\right|_{e=f}$. If $e \notin K$, then $K$ is a cover of $\mathcal{C}$, and if $e \in K$, then one of $K,(K \cup\{f\})-\{e\}$ is a cover of $\mathcal{C}$.

\footnotetext{
${ }^{2}$ In the literature, these clutters are referred to as degenerate projective planes. Adopting the terminology of [2], we refrain from doing so as these are not the only degenerate projective planes.
} 
(iii) $\tau\left(\left.\mathcal{C}\right|_{e=f}\right)=\tau(\mathcal{C})$.

(iv) If $\mathcal{C}$ does not pack, then neither does $\left.\mathcal{C}\right|_{e=f}$.

Proof. (i) is straightforward. (ii) If $e \notin K$, then clearly $K$ is still a cover of $\mathcal{C}$. Otherwise, when $e \in K$, it is clear that $K \cup\{f\}$ is a cover of $\mathcal{C}$. However, since $e, f$ are coexclusive elements of $\mathcal{C}$, it follows that one of $K,(K \cup\{f\})-\{e\}$ is also a cover of $\mathcal{C}$. (iii) It follows from (i) that $\tau(\mathcal{C}) \geq \tau\left(\left.\mathcal{C}\right|_{e=f}\right)$ and from (ii) that $\tau\left(\left.\mathcal{C}\right|_{e=f}\right) \geq \tau(\mathcal{C})$. (iv) is an immediate corollary of (iii), and that pairwise disjoint members of $\left.\mathcal{C}\right|_{e=f}$ correspond to pairwise disjoint members of $\mathcal{C}$.

Thus, identification reduces one non-packing clutter to a smaller one, so let us add this set-theoretic operation to the two minor operations of deletion and contraction. ${ }^{3}$ It is, however, possible for an ideal clutter to identify to a non-ideal one - for an example and a discussion see $\S 4.2$. As we will see in $\S 5.2$, the $Q_{r, t}$ 's do not have coexclusive elements. However, as briefly illustrated in $\S 4.1$, the dozen sporadic ideal mnp clutters found in [5] have coexclusive elements and therefore reduce further. As mentioned before, all these clutters have covering number 2 , and by using the additional operation of identification, we can show that mnp clutters with covering number 2 indeed have a key role:

Theorem 1.5. Let $\mathcal{C}$ be an ideal minimally non-packing clutter with coexclusive elements $e, f$. Then either

(i) $\left.\mathcal{C}\right|_{e=f}$ is another ideal minimally non-packing clutter, or

(ii) $\left.\mathcal{C}\right|_{e=f}$ is not minimally non-packing, and every minimally non-packing minor has covering number two.

This theorem is proved in $\S 4$. As for ideal mnp clutters with covering number 2 , we can show the following:

Theorem 1.6. Let $\mathcal{C}$ be an ideal minimally non-packing clutter without coexclusive elements, where $\tau(\mathcal{C})=2$. Then,

(i) the minimum covers partition $E(\mathcal{C})$,

(ii) the minimum size of a member is $\frac{|E(\mathcal{C})|}{2}$,

(iii) the members of minimum size form an ideal non-packing clutter $\mathcal{C}_{0}$ where $\tau\left(\mathcal{C}_{0}\right)=2$, and

(iv) $\mathcal{C}_{0}$ is a marginal cuboid.

This result is proved in $\S 5$. We will define marginal cuboids shortly. Statement (iv) provides a qualitative characterization explaining why the minimum members form an ideal non-packing clutter with covering number 2 . The experienced reader may notice the analogy between this result and Lehman's qualitative characterization of minimally non-ideal clutters.

\footnotetext{
${ }^{3} \mathrm{~A}$ clutter is non-packing if it does not pack.
} 


\subsection{Cuboids}

Take an integer $n \geq 1$. We will work over the $n$-dimensional hypercube $[0,1]^{n}$. Inequalities of the form $1 \geq x_{i} \geq 0, i \in[n]$ are called hypercube inequalities, and an inequality of the form

$$
\sum_{i \in I} x_{i}+\sum_{j \in J}\left(1-x_{j}\right) \geq 1 \quad \text { for disjoint } I, J \subseteq[n]
$$

is called a generalized set covering inequality. The latter forms quite a natural class of inequalities defined on the hypercube - one that does not distinguish between 0 and 1 . Now take a set $S \subseteq\{0,1\}^{n}$. Notice that the $2 n$-dimensional points

$$
\left(x_{1}, 1-x_{1}, x_{2}, 1-x_{2}, \ldots, x_{n}, 1-x_{n}\right) \in\{0,1\}^{2 n} \quad x \in S,
$$

when viewed as incidence vectors, correspond to a clutter over ground set $[2 n]$ whose members have size $n$; we call this clutter the cuboid of $S$. Whenever $S$ satisfies the following conditions,

(C1) for each $i \in[n],\left\{x \in S: x_{i}=0\right\} \neq \emptyset$ and $\left\{x \in S: x_{i}=1\right\} \neq \emptyset$,

(C2) if $x \in S$ then $\mathbf{1}-x \notin S$, and

(C3) the convex hull of $S$ can be described with hypercube and generalized set covering inequalities, we declare the cuboid of $S$ as marginal.

Proposition 1.7. Every marginal cuboid is an ideal non-packing clutter with covering number two.

This proposition is proved in $\S 5.3$. Looking back at Theorem 1.6, (iv) states that $\mathcal{C}_{0}$ is a marginal cuboid, so Proposition 1.7 explains why (iii) holds: (C1) is the reason the covering number of $\mathcal{C}_{0}$ is $2,(\mathrm{C} 2)$ is the reason it does not pack, and (C3) is the reason it is ideal. As the reader may have noticed, however, (C3) is an implicit condition and given a set of points, out of the three conditions, it is the most difficult to check. There is, however, a nice sufficient condition ensuring (C3) holds, found recently by Cornuéjols and Lee [6]. To this end, let $G_{n}$ denote the skeleton graph of the hypercube $[0,1]^{n}$ - this is a graph on vertices $\{0,1\}^{n}$ where two vertices are adjacent if they differ in exactly one coordinate.

Theorem 1.8 ([6]). Take an integer $n \geq 1$ and a subset $S \subseteq\{0,1\}^{n}$ where the vertex-induced subgraph $G_{n}\left[\{0,1\}^{n}-S\right]$ has maximum degree at most two. Then the convex hull of $S$ can be described with hypercube and generalized set covering inequalities.

Consider now the condition

(C3’) $G_{n}\left[\{0,1\}^{n}-S\right]$ has maximum degree at most two.

Theorem 1.8 states that (C3') implies (C3), and clearly this condition is much easier to check. (Not all marginal cuboids satisfy (C3'); see $\S 5.2$.) We now have a recipe for constructing ideal non-packing clutters with covering number 2: find a set of points satisfying $(\mathrm{C} 1),(\mathrm{C} 2)$ and $\left(\mathrm{C}^{\prime}\right)$ and take its cuboid. Using this recipe we can now construct infinitely many marginal cuboids. Three such marginal cuboids are described as follows: 
- $Q_{6}$ : a 6-dimensional marginal cuboid where $G_{3}\left[\{0,1\}^{3}-S\right]$ consists of 4 isolated vertices,

- $Q_{2,1}$ : an 8-dimensional marginal cuboid where $G_{4}\left[\{0,1\}^{4}-S\right]$ is a 6 -cycle and 2 isolated vertices,

- $Q_{10}$ : a 10-dimensional marginal cuboid where $G_{5}\left[\{0,1\}^{5}-S\right]$ is the vertex disjoint union of two 8-cycles.

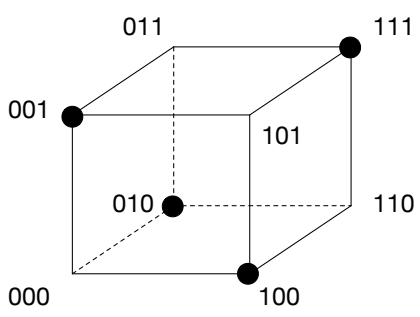

$Q_{6}$

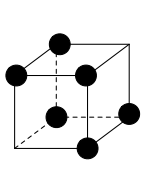

0

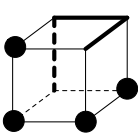

00

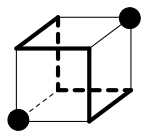

1

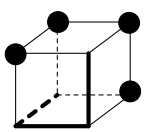

10

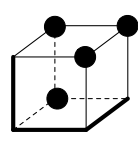

01

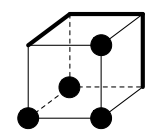

11

$Q_{2,1}$

$Q_{10}$

Figure 1: Bold vertices are the points in $S$.

These three clutters do not have coexclusive elements, and in fact, they are ideal, minimally non-packing. $Q_{6}$ was the first known example of an ideal mnp clutter, found by Lovász [13] (see also Seymour [19]), and has the least number of elements among all ideal non-packing clutters. $Q_{2,1}$ was discovered by Cornuéjols et al. [5]. $Q_{10}$, however, is a new ideal mnp clutter. ${ }^{4}$ The reader may now think that this easy-to-follow recipe would lead to more examples of ideal mnp clutters, but this is actually not the case:

Theorem 1.9. Take an integer $n \geq 1$ and a subset $S \subseteq\{0,1\}^{n}$ satisfying (C1), (C2) and (C3'). Then the marginal cuboid of $S$ has one of $Q_{6}, Q_{2,1}, Q_{10}$ as a cuboid minor. (In particular, this cuboid has one of $Q_{6}, Q_{2,1}, Q_{10}$ as a minor.)

A cuboid minor is a special form of minor that is defined in $\S 6$, where this theorem is also proved. Take integers $n, k \geq 1$ and a subset $S \subseteq\{0,1\}^{n}$. Motivated by Theorem 1.9, we say that the cuboid of $S$ has degree at most $k$ if the vertex-induced subgraph $G_{n}\left[\{0,1\}^{n}-S\right]$ has maximum degree at most $k$, and that the cuboid of $S$ has degree $k$ if it has degree at most $k$ and not $k-1$. For instance, cuboids satisfying (C3') have degree at most 2. However, in contrast to the ones of degree at most 2, cuboids of degree at least 3 are not necessarily ideal. For instance, the degree- 3 cuboid

$$
Q_{3}:=\{\{1,4,6\},\{2,3,6\},\{2,4,5\}\}
$$

of $\{100,010,001\} \subseteq\{0,1\}^{3}$ is non-ideal as it has a $\Delta_{3}$ minor, obtained after contracting elements $1,3,5$ (and relabeling $2,4,6$ by $1,2,3)$.

Theorem 1.10. Take integers $n, k \geq 1$ and a subset of $S \subseteq\{0,1\}^{n}$ whose cuboid $\mathcal{C}$ has degree at most $k$. Then the following statements hold:

\footnotetext{
${ }^{4}$ The fact that $Q_{10}$ is mnp can be verified using a computer.
} 
(i) If $\mathcal{C}$ is non-packing and $n \geq 4 k+2$, then $\mathcal{C}$ has one of $Q_{3}, Q_{6}$ as a cuboid minor.

(ii) If $\mathcal{C}$ has a non-packing minor, then it has one with at most $8 k+2$ elements.

(iii) If $\mathcal{C}$ is non-ideal, then every minimally non-ideal minor has at most $k$ elements.

This result is proved in $\S 6$. Notice that Theorem 1.10 (i) extends Theorem 1.9 to bounded degree cuboids. Moreover, since every non-ideal clutter has at least 3 elements, Theorem 1.10 (iii) alternatively shows that cuboids of degree at most 2 are always ideal.

\section{Delta minors}

Here we provide a tool for spotting delta minors. This tool will help us prove Theorems 1.5 and 1.6.

Recall that for $n \geq 3, \Delta_{n}=\{\{1,2\},\{1,3\}, \ldots,\{1, n\},\{2,3, \ldots, n\}\}$. Observe that $b\left(\Delta_{n}\right)=\Delta_{n}$. This attribute will be of great help when looking for delta minors. Indeed, if $\mathcal{C}^{\prime}:=\mathcal{C} \backslash I / J$ is a minor of $\mathcal{C}$, then $b\left(\mathcal{C}^{\prime}\right)$ is a minor of $b(\mathcal{C})$ as $b\left(\mathcal{C}^{\prime}\right)=b(\mathcal{C} \backslash I / J)=b(\mathcal{C}) / I \backslash J$. In particular, since $b\left(\Delta_{n}\right)=\Delta_{n}$, a delta minor in the blocker guarantees a delta minor in the clutter itself. We will take advantage of this observation.

To describe the tool, we need the following terminology. Let $\mathcal{C}$ be a clutter, fix an element $e$, and take a member $C$ not containing $e$. We say $C$ is e-redundant if for a member $C^{\prime}$ containing $e$, we have that $C^{\prime}-\{e\} \subsetneq$ $C$. Member $C^{\prime}$ is called a cause of redundancy. Equivalently, a member is $e$-redundant if it becomes redundant after element $e$ is contracted (hence our choice of terminology). We say $C$ is doubly e-redundant if it is $e$ redundant with at least two distinct causes.

Theorem 2.1. Let $\mathcal{C}$ be a clutter over ground set $E$, and fix an element $e \in E$. If there is a doubly e-redundant member, then there is a delta minor using element e that can be found in time polynomial in $|E|,|\mathcal{C}|$.

Proof. We may assume that $\mathcal{C}$, over ground set $E$, is minor-minimal with respect to having a doubly $e$-redundant member. We will show that $\mathcal{C}$ is in fact a delta. Suppose member $C$ is doubly $e$-redundant, that is, $e \notin C$ and for two distinct members $C_{1}, C_{2}$ containing $e$, we have that $C_{1}-\{e\} \subsetneq C$ and $C_{2}-\{e\} \subsetneq C$. Note that the minimality assumption implies

(1) $C_{1} \cap C_{2}=\{e\}$,

because for $I:=C_{1} \cap C_{2}-\{e\}$, the minor $\mathcal{C} / I$ has $C-I$ as a doubly $e$-redundant member with causes $C_{1}-I$ and $C_{2}-I$. The minimality assumption also implies that

(2) $\{e\} \cup C=E$,

because for $J:=E-(\{e\} \cup C), \mathcal{C} \backslash J$ has $C$ as a doubly $e$-redundant member. Next, we claim that

(3) $\left|C_{1}\right|=\left|C_{2}\right|=2$. 
To see this, suppose for a contradiction that one of $C_{1}, C_{2}$, say $C_{1}$, has size at least 3 . Pick an element $h \in$ $C_{1}-\{e\}$, and note that by (1), $h \notin C_{2}$. Consider the minor $\mathcal{C}^{\prime}:=\mathcal{C} / h$, for which $C_{1}^{\prime}:=C_{1}-\{h\}$ and $C^{\prime}:=C-\{h\}$ are still members. Notice that $C_{2}$ contains a member $C_{2}^{\prime}$ of $\mathcal{C}^{\prime}$, for which it is easy to see that $e \in C_{2}^{\prime}$ and $C_{2}^{\prime} \neq\{e\}$. But now $\mathcal{C}^{\prime}$ has $C^{\prime}$ as a doubly $e$-redundant member with causes $C_{1}^{\prime}, C_{2}^{\prime}$, a contradiction to our minimality assumption. This proves (3). Now let $X:=\{f \in E:\{e, f\}$ is a member $\}$. So $|X| \geq 2$ by (3), and $X \subseteq C$ by (2). Our last claim is that

$$
\text { (4) } X=C \text {. }
$$

For if not, pick an element $h \in C-X$, and note that $C-\{h\}$ is doubly $e$-redundant for $\mathcal{C} / h$ with causes $C_{1}, C_{2}$, contradicting the minimality assumption. Thus, $X=C$. It now easily follows from (2) and the definition of $X$ that $\mathcal{C}$ is a delta, as promised.

This result has the following useful consequence:

Corollary 2.2. Let $\mathcal{C}$ be a clutter without a delta minor, and take distinct elements $e, f, g$. If $\{e, f\},\{e, g\}$ are members, then $f$ and $g$ are exclusive elements. Similarly, if $\{e, f\},\{e, g\}$ are minimal covers, then $f$ and $g$ are coexclusive elements.

Proof. Assume that $\{e, f\},\{e, g\}$ are members. If there is a member $C$ containing $f$ and $g$, then $C$ is doubly $e$-redundant with causes $\{e, f\}$ and $\{e, g\}$, so by Theorem $2.1, \mathcal{C}$ has a delta minor, which is not the case. Thus, every member contains at most one of $f$ and $g$, i.e. these elements are exclusive.

Observe that exclusive (resp. coexclusive) elements of a clutter remain exclusive (resp. coexclusive) in every minor where the elements are present. This fact will be useful in the following consequence of the corollary above that we cannot resist to include.

Theorem 2.3. There is an algorithm that given a clutter $\mathcal{C}$ over ground set $E$ finds in time polynomial in $|E|,|\mathcal{C}|$ a delta minor or certify that none exists.

Proof. We claim that the following statements are equivalent:

(i) $\mathcal{C}$ does not have a delta minor,

(ii) for all distinct members $C_{1}, C_{2}$ with $C_{1} \cap C_{2} \neq \emptyset$ and for all elements $e, f, g$ with $e \in C_{1} \cap C_{2}, f \in$ $C_{1}-C_{2}, g \in C_{2}-C_{1}$, the following holds: for $X:=\left(C_{1} \cup C_{2}\right)-\{e, f, g\}$ and $\mathcal{C}^{\prime}:=\mathcal{C} / X$, either $\{e, f\} \notin \mathcal{C}^{\prime}$ or $\{e, g\} \notin \mathcal{C}^{\prime}$ or $f, g$ are exclusive elements of $\mathcal{C}^{\prime}$.

(ii) $\Rightarrow$ (i): Assume that (i) does not hold. Suppose $\mathcal{C}$ has a delta minor obtained after deleting $I \subseteq E$ and contracting $J \subseteq E$. Pick elements $e, f, g \in E-(I \cup J)$ such that $\{e, f\},\{e, g\}$ are members of the delta minor. Notice that $f, g$ are not exclusive elements in the delta minor, and so they are not exclusive in $\mathcal{C}$. Let $C_{1}, C_{2}$ be members of $\mathcal{C}$ such that $\{e, f\} \subseteq C_{1} \subseteq\{e, f\} \cup J$ and $\{e, g\} \subseteq C_{2} \subseteq\{e, g\} \cup J$. It can be readily checked that $C_{1}, C_{2}$ and $e, f, g$ do not satisfy (ii). Thus, (ii) does not hold. (i) $\Rightarrow$ (ii): Assume that (i) holds. 
Take $C_{1}, C_{2}, e, f, g, X, \mathcal{C}^{\prime}$ as in (ii) where $\{e, f\} \in \mathcal{C}^{\prime}$ and $\{e, g\} \in \mathcal{C}^{\prime}$. Since $\mathcal{C}$ has no delta minor, neither does $\mathcal{C}^{\prime}$, so by Corollary 2.2, $f$ and $g$ are exclusive elements of $\mathcal{C}^{\prime}$, so (ii) holds. Hence, (i) and (ii) are equivalent. Since (ii) may be verified in time polynomial in $|E|,|\mathcal{C}|$, and if (ii) does not hold, a delta minor can be found in time polynomial in $|E|,|\mathcal{C}|$ using Theorem 2.1 , we can find a delta minor or certify that none exists in time polynomial in $|E|,|\mathcal{C}|$.

\section{Coexclusive elements}

Here we prove Theorem 1.2, discuss coexclusive elements and their relevance to ideal non-packing clutters. The results in this section will help us in proving Theorems 1.5 and 1.6, as well as Proposition 1.7.

Let us first prove Theorem 1.2, which provides two characterizations of coexclusive elements. A few observations before the proof: for a clutter $\mathcal{C}$, the integral extreme points of $Q(\mathcal{C})$ are precisely the incidence vectors of the minimal covers, and in general, every extreme point of $Q(\mathcal{C})$ is bounded above by 1 .

Proof of Theorem 1.2. (i) $\Rightarrow$ (ii): Suppose $e, f$ are coexclusive elements of clutter $\mathcal{C}$. Take members $C_{e}, C_{f}$ where $C_{e} \cap\{e, f\}=\{e\}$ and $C_{f} \cap\{e, f\}=\{f\}$. We will show that $C_{e} \cup C_{f}-\{e, f\}$ contains a member, thereby proving (ii). Suppose otherwise. Then the complement of $C_{e} \cup C_{f}-\{e, f\}$ is a cover, so it contains a minimal cover $B$. Since $B \cap C_{e} \neq \emptyset$ and $B \cap C_{f} \neq \emptyset$, we get that $\{e, f\} \subseteq B$, contradicting the fact that $e, f$ are coexclusive. (ii) $\Rightarrow$ (iii): Take an extreme point $x^{\star}$ of $Q(\mathcal{C})$. We will show that $x_{e}^{\star}+x_{f}^{\star} \leq 1$, proving (iii). If $x_{e}^{\star}=0$ or $x_{f}^{\star}=0$, then clearly $x_{e}^{\star}+x_{f}^{\star} \leq 1$. Otherwise, there is a member $C_{e}$ with $e \in C_{e}$ and a member $C_{f}$ with $f \in C_{f}$ such that $x^{\star}\left(C_{e}\right)=x^{\star}\left(C_{f}\right)=1$. If $\{e, f\} \subseteq C_{e}$, then $x_{e}^{\star}+x_{f}^{\star} \leq x^{\star}\left(C_{e}\right)=1$. We may therefore assume that $C_{e} \cap\{e, f\}=\{e\}$ and, similarly, $C_{f} \cap\{e, f\}=\{f\}$. It now follows from (ii) that there is a member $C \subseteq C_{e} \cup C_{f}-\{e, f\}$. Then

$$
x_{e}^{\star}+x_{f}^{\star}+1 \leq x_{e}^{\star}+x_{f}^{\star}+x^{\star}(C) \leq x^{\star}\left(C_{e}\right)+x^{\star}\left(C_{f}\right)=2,
$$

proving (iii). (iii) $\Rightarrow$ (i): Since the incidence vector of every minimal cover $B$ is an extreme point $x^{\star}$ of $Q(\mathcal{C})$, we get from $x_{e}^{\star}+x_{f}^{\star} \leq 1$ that $B$ contains at most one $e, f$. So $e, f$ are coexclusive, proving (i).

That coexclusive elements have different characterizations only stresses their importance and relevance to clutter theory. Abdi, Fukasawa and Sanità [2] introduced and studied opposite elements, a pair of coexclusive elements that are also exclusive. They observed how opposite elements show up in clutters arising from bidirected graphs, and how identification of such elements is a natural operation. Although coexclusive elements do not possess as much of a local structure, they still do turn up when dealing with directed graphs - let us elaborate.

Let $D=(V, A)$ be a directed graph. A dicut is a cut whose arcs are oriented in unison from the inside shore to the outside shore, that is, it is of the form $\delta^{+}(U)$ where $\delta^{-}(U)=\emptyset$, for some $\emptyset \neq U \subsetneq V$. A dijoin is a cover of the clutter of minimal dicuts; equivalently, a dijoin is an arc subset whose contraction makes the directed graph strongly connected. The Lucchesi-Younger theorem [14] implies that the clutter of minimal dijoins is an ideal clutter. Although Woodall [20] conjectures that this clutter packs, it is known that this clutter may have 
non-packing minors [16]. If history is any indication, dijoin clutters are difficult to deal with. So the reader may find it interesting (or perhaps disappointing) that dijoin clutters have many pairs of coexclusive arcs: a pair of arcs on a directed path can never be used together in a dicut and are therefore coexclusive. Moreover, an identification of a dijoin clutter is not necessarily another dijoin clutter - so identification seems to collapse dijoin clutters.

Not only coexclusive elements, but exclusive elements are also relevant. Our just proven characterization of coexclusive elements has the following consequence, a crucial tool for Theorem 1.6 and Proposition 1.7.

Lemma 3.1. Take a clutter $\mathcal{C}$ over ground set $E=\left\{e_{1}, f_{1}, \ldots, e_{n}, f_{n}\right\}$, where for each $i \in[n],\left\{e_{i}, f_{i}\right\}$ intersects every member exactly once. Then the following statements are equivalent:

(i) $\mathcal{C}$ is ideal,

(ii) $\operatorname{conv}\left\{\chi_{C}: C \in \mathcal{C}\right\}=Q(b(\mathcal{C})) \cap\left\{x: x_{e_{i}}+x_{f_{i}}=1 \forall i \in[n]\right\}$.

Here, $\chi_{C} \subseteq\{0,1\}^{E}$ is the incidence vector of $C \subseteq E$. Moreover, since every member contains exactly one of $e_{i}, f_{i}, \subseteq$ always holds in (ii), so $\supseteq$ is the main restriction of (ii).

Proof. (i) $\Rightarrow$ (ii): The inclusion $\subseteq$ holds trivially. Let us prove the reverse inclusion $\supseteq$. Since $\mathcal{C}$ is ideal, we get that $b(\mathcal{C})$ is ideal, so

$$
Q(b(\mathcal{C}))=\text { dominant of } \operatorname{conv}\left\{\chi_{C}: C \in \mathcal{C}\right\}=\operatorname{conv}\left\{\chi_{C}: C \in \mathcal{C}\right\}+\mathbb{R}_{+}^{E}
$$

It is easy to see that this equation implies the reverse inclusion. (ii) $\Rightarrow(\mathbf{i})$ : We will show that $b(\mathcal{C})$ is ideal, implying in turn that $\mathcal{C}=b(b(\mathcal{C}))$ is ideal, thereby proving (i). To this end, let $x^{\star}$ be an extreme point of $Q(b(\mathcal{C}))$. It suffices to show that $x^{\star}$ is integral. Since $\left\{e_{i}, f_{i}\right\}$ is a cover, we get that $x_{e_{i}}^{\star}+x_{f_{i}}^{\star} \geq 1$. Moreover, since $e_{i}, f_{i}$ are exclusive in $\mathcal{C}$, they are coexclusive in $b(\mathcal{C})$, so by Theorem 1.2 (iii), $x_{e_{i}}^{\star}+x_{f_{i}}^{\star} \leq 1$. So for each $i \in[n], x_{e_{i}}^{\star}+x_{f_{i}}^{\star}=1$, implying in turn by (ii) that $x^{\star} \in \operatorname{conv}\left\{\chi_{C}: C \in \mathcal{C}\right\}$. Since $x^{\star}$ is an extreme point, it must be one of the incidence vectors and hence integral, as required.

This lemma also follows from [9] and [15].

\section{Property preserving identifications}

We say that a clutter has the packing property if every minor of it (including itself) packs. We characterize when identification preserves the packing property and when it preserves idealness. Using these results, we will prove Theorem 1.5. We will need the following observation:

Remark 4.1. Let $\mathcal{C}$ be a clutter and take coexclusive elements $e, f$. Then for subsets $I, J \subseteq E(\mathcal{C})-\{e, f\}$ that are disjoint, the following statements hold:

(i) e, f are coexclusive elements of $\mathcal{C} / I \backslash J$,

(ii) $\left.\mathcal{C}\right|_{e=f} / I \backslash J=\left.(\mathcal{C} / I \backslash J)\right|_{e=f}$ 


\subsection{The packing property}

We begin with the following:

Theorem 4.2. Take a clutter $\mathcal{C}$ that has the packing property, with coexclusive elements e, $f$. The following statements are equivalent:

(i) $\left.\mathcal{C}\right|_{e=f}$ has the packing property,

(ii) every minor of $\left.\mathcal{C}\right|_{e=f}$ with covering number at least 2 , has two disjoint members,

(iii) for all members $C_{e}, C_{f}$ of $\mathcal{C}$ where $C_{e} \cap\{e, f\}=\{e\}$ and $C_{f} \cap\{e, f\}=\{f\}$, there are members $C$, $C^{\prime}$ such that

$$
C \cap\{e, f\}=\emptyset, \quad C \cap C^{\prime} \subseteq C_{e} \cap C_{f} \quad \text { and } \quad C \cup C^{\prime} \subseteq C_{e} \cup C_{f}
$$

Proof. (i) $\Rightarrow$ (ii): This follows from the definition of the packing property. (ii) $\Rightarrow$ (iii): Let $I:=C_{e} \cap C_{f}$ and $J:=E(\mathcal{C})-\left(C_{e} \cup C_{f}\right)$. Consider the minor $\mathcal{C}^{\prime}:=\mathcal{C} / I \backslash J$. Since $C_{e}-I$ and $C_{f}-I$ are disjoint members of $\mathcal{C}^{\prime}$, we get that $\tau\left(\mathcal{C}^{\prime}\right) \geq 2$. By Remark 4.1, e and $f$ are still coexclusive elements in $\mathcal{C}^{\prime}$ whose corresponding identification is $\left.\mathcal{C}\right|_{e=f} / I \backslash J$. So by Remark 1.4 (iii) we get that $\tau\left(\left.\mathcal{C}\right|_{e=f} / I \backslash J\right)=\tau\left(\mathcal{C}^{\prime}\right) \geq 2$. Now (ii) implies the existence of two disjoint members in $\left.\mathcal{C}\right|_{e=f} / I \backslash J$ - these disjoint members will correspond to members $C, C^{\prime}$ of $\mathcal{C}$ satisfying (iii). (iii) $\Rightarrow$ (i): Since (iii) is a minor-closed property, it suffices to show that $\left.\mathcal{C}\right|_{e=f}$ packs. To this end, let $\tau:=\tau(\mathcal{C})=\tau\left(\left.\mathcal{C}\right|_{e=f}\right.$ ) (recall Remark 1.4 (iii)). Since $\mathcal{C}$ packs, it has $\tau$ pairwise disjoint members $C_{1}, \ldots, C_{\tau}$, where $C_{i} \cap\{e, f\}=\emptyset$ for each $i \in\{3, \ldots, \tau\}$. If one of $C_{1}, C_{2}$ is also disjoint from $\{e, f\}$, then these members yield the desired packing of size $\tau$ in $\left.\mathcal{C}\right|_{e=f}$. We may therefore assume that $C_{1} \cap\{e, f\}=\{e\}$ and $C_{2} \cap\{e, f\}=\{f\}$. It then follows from (iii) that there are disjoint members $C, C^{\prime}$ contained in $C_{1} \cup C_{2}$ such that $C \cap\{e, f\}=\emptyset$. It is easy to see that $C, C^{\prime}, C_{3}, \ldots, C_{\tau}$ gives rise to a packing of size $\tau$ in $\left.\mathcal{C}\right|_{e=f}$, as required.

This characterization has the following corollary that we will use:

Corollary 4.3. Take a minimally non-packing clutter $\mathcal{C}$ with coexclusive elements $e, f$. Then either

(i) $\left.\mathcal{C}\right|_{e=f}$ is minimally non-packing, or

(ii) every minimally non-packing minor of $\left.\mathcal{C}\right|_{e=f}$ has covering number 2.

Proof. Suppose (i) is not the case, that is, $\left.\mathcal{C}\right|_{e=f}$ is not mnp. We will prove that (ii) holds. Let $\left.\mathcal{C}\right|_{e=f} \backslash I / J$ be an mnp minor of $\left.\mathcal{C}\right|_{e=f}$ for disjoint element subsets $I, J$ where $I \cup J \neq \emptyset$. Since $\left.\mathcal{C}\right|_{e=f} \backslash I / J$ is not a minor of $\mathcal{C}$, we have that $(I \cup J) \cap\{e, f\}=\emptyset$. Consider the clutter $\mathcal{C}^{\prime}:=\mathcal{C} \backslash I / J$. Being a proper minor of the mnp $\mathcal{C}$, we see that $\mathcal{C}^{\prime}$ has the packing property. Its identification $\left.\mathcal{C}^{\prime}\right|_{e=f}=\left.\mathcal{C}\right|_{e=f} \backslash I / J$ however, is mnp (the equation follows from Remark 4.1). Thus, Theorem 4.2 implies that a minor of $\left.\mathcal{C}^{\prime}\right|_{e=f}$ with covering number at least 2 
does not have two disjoint members. Since every proper minor of $\left.\mathcal{C}^{\prime}\right|_{e=f}$ packs, the clutter $\left.\mathcal{C}^{\prime}\right|_{e=f}$ itself does not have two disjoint members. This immediately implies that

$$
\tau\left(\left.\mathcal{C}\right|_{e=f} \backslash I / J\right)=\tau\left(\left.\mathcal{C}^{\prime}\right|_{e=f}\right)=2,
$$

(otherwise delete any element of $\left.\mathcal{C}^{\prime}\right|_{e=f}$ to obtain another non-packing minor), as required.

An infinite class of examples satisfying Corollary 4.3 (ii) is obtained as follows. Take an odd integer $n \geq 5$. An odd hole of dimension $n$, denoted $\mathcal{C}_{n}^{2}$, is the clutter over ground set $\{1,2,3, \ldots, n\}$ whose members are

$$
\mathcal{C}_{n}^{2}=\{\{1,2\},\{2,3\}, \ldots,\{n-1, n\},\{n, 1\}\}
$$

Let $\mathcal{C}_{3}^{2}:=\Delta_{3}$. It is well-known that $\mathcal{C}_{3}^{2}, \mathcal{C}_{5}^{2}, \mathcal{C}_{7}^{2}, \ldots$ and their blockers $b\left(\mathcal{C}_{3}^{2}\right), b\left(\mathcal{C}_{5}^{2}\right), b\left(\mathcal{C}_{7}^{2}\right), \ldots$ are (non-ideal) mnp clutters. Notice that $n-1,1$ are coexclusive in $b\left(\mathcal{C}_{n}^{2}\right)$, and that $\left.b\left(\mathcal{C}_{n}^{2}\right)\right|_{n-1=1}$ has $b\left(\mathcal{C}_{n-2}^{2}\right)$ as its only mnp minor, whose covering number is 2 , agreeing with Corollary 4.3 (ii).

As mentioned in the introduction, other than the $Q_{r, t}$ 's, a dozen examples of (ideal) mnp clutters were introduced in [5]. All of these clutters have a pair of coexclusive elements whose identification agrees with Corollary 4.3 (i). For instance, they discovered the mnp clutter whose incidence matrix is $M$ as displayed below (for readability's sake, the zeros are removed). Using Theorem 1.2 (ii), it is easily seen that the first and second columns correspond to coexclusive elements, and that identifying them leads to another mnp clutter $Q_{6} \otimes 1$ they found; the second and third columns here are again coexclusive and identifying them gives the mnp $Q_{6}$, agreeing with Corollary 4.3 (i). What is more, in these examples, not only is $\left.\mathcal{C}\right|_{e=f} \mathrm{mnp}$, but it is also ideal. This is not a coincidence, and as Theorem 1.5 claims, whenever $\mathcal{C}$ is ideal mnp and $\left.\mathcal{C}\right|_{e=f}$ is mnp, then $\left.\mathcal{C}\right|_{e=f}$ is also ideal.

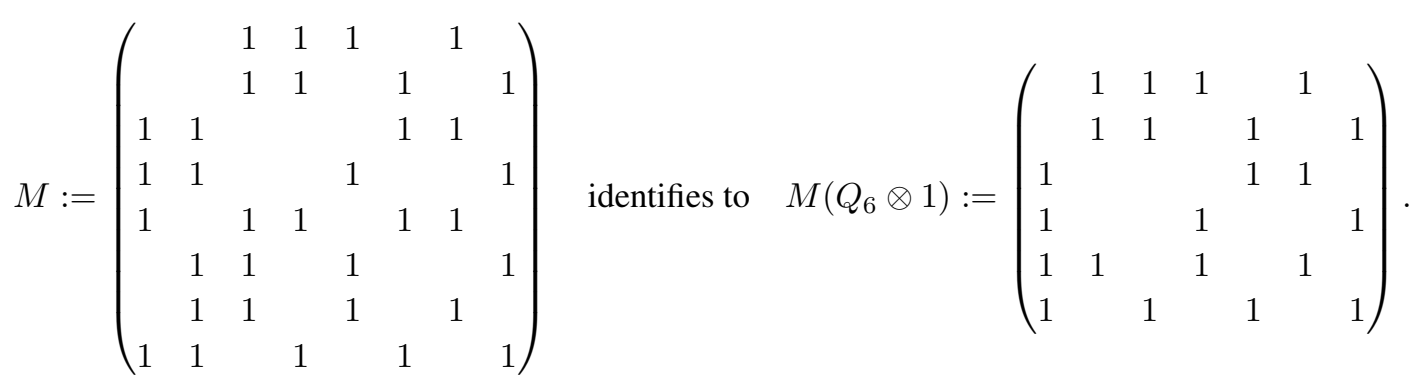

To prove this however, we will need a fractional analogue of Theorem 4.2.

\subsection{Idealness}

Let $\mathcal{C}$ be a clutter over ground set $E$. Consider the pair of dual linear programs

$$
\text { (P) }\left\{\begin{array} { l l } 
{ \operatorname { m i n } } & { \sum ( x _ { g } : g \in E ) } \\
{ \text { s.t. } } & { x ( C ) \geq 1 \quad \forall C \in \mathcal { C } } \\
{ } & { x \geq \mathbf { 0 } }
\end{array} \quad \text { (D) } \left\{\begin{array}{ll}
\max & \sum\left(y_{C}: C \in \mathcal{C}\right) \\
\text { s.t. } & \sum\left(y_{C}: C \in \mathcal{C}, g \in C\right) \leq 1 \quad \forall g \in E \\
& y \geq \mathbf{0} .
\end{array}\right.\right.
$$

Observe that $Q(\mathcal{C})$ is the set of feasible solutions to $(\mathrm{P})$. A feasible solution to the dual program (D) is called a fractional packing and its value is the objective value of the solution. When $\mathcal{C}$ is ideal, basic polyhedral 
theory dictates that a minimum cover yields an optimal solution to (P), and thus by Strong Duality, there exists a fractional packing of value $\tau(\mathcal{C})$ ([4], Theorems 3.7 and 4.1).

Now take coexclusive elements $e, f$ and members $C_{e}, C_{f}$ such that

$(\star) C_{e} \cap\{e, f\}=\{e\}$ and $C_{f} \cap\{e, f\}=\{f\}$.

A fractional disentangling of $C_{e}$ and $C_{f}$ is a vector $y \in \mathbb{R}_{+}^{\mathcal{C}}$ where

- $\sum\left(y_{C}: C \in \mathcal{C}\right)=2$,

- $\sum\left(y_{C}: C \cap\{e, f\} \neq \emptyset\right) \leq 1$, and

- for each element $g$,

$$
\sum\left(y_{C}: g \in C\right) \leq \begin{cases}0 & \text { if } g \notin C_{e} \cup C_{f} \\ 1 & \text { if } g \in C_{e} \triangle C_{f}=\left(C_{e} \cup C_{f}\right)-\left(C_{e} \cap C_{f}\right) \\ 2 & \text { if } g \in C_{e} \cap C_{f} .\end{cases}
$$

Notice that a fractional disentangling is the fractional analogue of the two members in part (iii) of Theorem 4.2. We are now ready to state the fractional analogue of this theorem:

Theorem 4.4. Take an ideal clutter $\mathcal{C}$ with coexclusive elements e, $f$. The following statements are equivalent:

(i) $\left.\mathcal{C}\right|_{e=f}$ is ideal,

(ii) every minor of $\left.\mathcal{C}\right|_{e=f}$ with covering number at least 2 , has a fractional packing of value 2 ,

(iii) all members $C_{e}, C_{f}$ of $\mathcal{C}$ satisfying $(\star)$ have a fractional disentangling,

(iv) for all members $C_{e}, C_{f}$ of $\mathcal{C}$ satisfying ( $\star$, the inequality

$$
x\left(C_{e}-\{e\}\right)+x\left(C_{f}-\{f\}\right)+x_{e} \geq 2
$$

is valid for $Q\left(\left.\mathcal{C}\right|_{e=f}\right)$.

Proof. Let $E:=E(\mathcal{C})$. (i) $\Rightarrow$ (ii): Notice that every minor of $\left.\mathcal{C}\right|_{e=f}$ is ideal, so (ii) follows immediately from our discussion above on fractional packings. (ii) $\Rightarrow$ (iii): As in Theorem 4.2, let $I:=C_{e} \cap C_{f}$ and $J:=E-\left(C_{e} \cup C_{f}\right)$. Consider the minor $\mathcal{C}^{\prime}:=\mathcal{C} / I \backslash J$. Since $C_{e}-I$ and $C_{f}-I$ are disjoint members of $\mathcal{C}^{\prime}$, we get that $\tau\left(\mathcal{C}^{\prime}\right) \geq 2$. By Remark 4.1,e and $f$ are still coexclusive in $\mathcal{C}^{\prime}$ and the corresponding identification is $\left.\mathcal{C}^{\prime}\right|_{e=f}=\left.\mathcal{C}\right|_{e=f} / I \backslash J$. It therefore follows from Remark 1.4 (iii) that $\tau\left(\left.\mathcal{C}^{\prime}\right|_{e=f}\right)=\tau\left(\mathcal{C}^{\prime}\right) \geq 2$. Now (ii) implies the existence of a fractional packing $y \in \mathbb{R}_{+}^{\left.\mathcal{C}^{\prime}\right|_{e=f}}$ of value 2 . Consider the natural extension of $y$ to $\mathbb{R}_{+}^{\mathcal{C}}$ where members of $\mathcal{C}$ present in the identified minor $\left.\mathcal{C}^{\prime}\right|_{e=f}$ are assigned the same value as before, and all the other members are assigned 0 . It can be readily checked that this natural extension is a fractional disentangling 
of $C_{e}$ and $C_{f}$. (iii) $\Rightarrow$ (iv): Let $y \in \mathbb{R}_{+}^{\mathcal{C}}$ be a fractional disentangling of $C_{e}$ and $C_{f}$. Since the members of $\mathcal{C}$ are in correspondence with the sets in the collection

$$
\widehat{\left.\mathcal{C}\right|_{e=f}}:=\{C: f \notin C \in \mathcal{C}\} \cup\{(C \cup\{e\})-\{f\}: f \in C \in \mathcal{C}\}
$$

we may regard $y$ as a vector in $\mathbb{R}_{+}^{\widehat{\left.\mathcal{C}\right|_{e=f}}}$. Observe further that $\left.\mathcal{C}\right|_{e=f}$ consists of the minimal sets in $\widehat{\left.\mathcal{C}\right|_{e=f}}$. As a result, for every $x \in Q\left(\left.\mathcal{C}\right|_{e=f}\right)$, we have

$$
\begin{aligned}
0 \leq & \sum_{C \in \widehat{\left.\mathcal{C}\right|_{e=f}}} y_{C}(x(C)-1) \\
= & \sum_{C \in \widehat{\left.\mathcal{C}\right|_{e=f}}} y_{C} \cdot x(C)-2 \\
= & \sum_{g \in E-\{f\}} x_{g}\left[\sum\left(y_{C}: g \in C \in \widehat{\left.\mathcal{C}\right|_{e=f}}\right)\right]-2 \\
= & x_{e}\left[\sum\left(y_{C}: e \in C \in \widehat{\left.\mathcal{C}\right|_{e=f}}\right)\right]+\sum_{g \notin C_{e} \cup C_{f}} x_{g}\left[\sum_{g \in C}\left(y_{C}: g \in C \in \widehat{\left.\mathcal{C}\right|_{e=f}}\right)\right] \\
& +\sum_{g \in C_{e} \Delta C_{f}-\{e, f\}} x_{g}\left[\sum\left(y_{C}: g \in C \in \widehat{\left.\mathcal{C}\right|_{e=f}}\right)\right]+\sum_{g \in C_{e} \cap C_{f}} x_{g}\left[\sum\left(y_{C}: g \in C \in \widehat{\left.\mathcal{C}\right|_{e=f}}\right)\right]-2 \\
\leq & x_{e} \cdot 1+\sum_{g \notin C_{e} \cup C_{f}} x_{g} \cdot 0+\sum_{g \in C_{e} \Delta C_{f}-\{e, f\}} x_{g} \cdot 1+\sum_{g \in C_{e} \cap C_{f}} x_{g} \cdot 2-2 \\
= & x\left(C_{e}-\{e\}\right)+x\left(C_{f}-\{f\}\right)+x_{e}-2,
\end{aligned}
$$

where the second to last line follows from the fact that $y$ is a fractional disentangling. The last equation proves (iv). (iv) $\Rightarrow$ (i): Since $\mathcal{C}$ is ideal, the linear system

$$
\begin{aligned}
& x(C-\{e, f\})+z(C \cap\{e, f\}) \geq 1 \quad \forall C \in \mathcal{C} \\
& x_{g} \geq 0 \quad \forall g \in E-\{e, f\} \\
& z_{e}, z_{f} \geq 0
\end{aligned}
$$

describes the dominant of $\operatorname{conv}\left\{\chi_{B}: B \in b(\mathcal{C})\right\}$. We now add a new variable $x_{e}$ with the additional linear constraint $x_{e}=z_{e}+z_{f}$. Remark 1.4 implies that the dominant of $\operatorname{conv}\left\{\chi_{B^{\prime}}: B^{\prime} \in b\left(\left.\mathcal{C}\right|_{e=f}\right)\right\}$ can be described by this new linear system after eliminating variables $z_{e}$ and $z_{f}$. After applying the Fourier-Motzkin Elimination method to do so, we get that the dominant of $\operatorname{conv}\left\{\chi_{B^{\prime}}: B^{\prime} \in b\left(\left.\mathcal{C}\right|_{e=f}\right)\right\}$ is described by

$$
\begin{aligned}
x(C) \geq 1 & \left.\forall C \in \mathcal{C}\right|_{e=f} \\
x_{g} \geq 0 & \forall g \in E-\{f\} \\
x\left(C_{e}-\{e\}\right)+x\left(C_{f}-\{f\}\right)+x_{e} \geq 2 & \text { for all } C_{e}, C_{f} \text { satisfying }(\star) .
\end{aligned}
$$

However, it follows from (iv) that the last line of inequalities are all redundant, implying in turn that $Q\left(\left.\mathcal{C}\right|_{e=f}\right)$, which is the dominant of $\operatorname{conv}\left\{\chi_{B^{\prime}}: B^{\prime} \in b\left(\left.\mathcal{C}\right|_{e=f}\right)\right\}$, is an integral polyhedron, thereby proving (i). 
This characterization has the following consequence:

Corollary 4.5. Take an ideal clutter $\mathcal{C}$ with coexclusive elements $e, f$. If $\left.\mathcal{C}\right|_{e=f}$ is minimally non-ideal, then $\tau(\mathcal{C})=\tau\left(\left.\mathcal{C}\right|_{e=f}\right)=2$.

Proof. Suppose $\left.\mathcal{C}\right|_{e=f}$ is mni. It then follows from Theorem 4.4 that a minor of $\left.\mathcal{C}\right|_{e=f}$ with covering number at least 2 has no fractional packing of value 2 . As every proper minor of it is ideal, we get that $\left.\mathcal{C}\right|_{e=f}$ itself has no fractional packing of value 2. This implies that $\tau\left(\left.\mathcal{C}\right|_{e=f}\right)=2$, as required. (For if not, delete any element and since the covering number is at least 2 , this minor has a fractional packing of value 2 , corresponding to a fractional packing of the same value in $\left.\mathcal{C}\right|_{e=f}$, which is not the case.)

For example, the ideal clutter $\mathbb{P}_{4}=\{\{1,2\},\{2,3\},\{3,4\}\}$ has coexclusive elements 1,4 and identifying them gives the mni $\mathcal{C}_{3}^{2}=\Delta_{3}$, whose covering number is 2 .

\subsection{Proof of Theorem 1.5}

A straightforward corollary of Lehman's result on minimally non-ideal clutters [12] is the following:

Theorem 4.6 (see $[17,11])$. Suppose $\mathcal{C}$ is a minimally non-ideal clutter with n elements, where $\tau(\mathcal{C})=2$. Then either

(i) $\mathcal{C}=\Delta_{n}$, or

(ii) the minimum covers of $\mathcal{C}$ form an odd hole of dimension $n$.

Moreover, each minimum cover is contained in a member.

Using this result, we can provide the last ingredient for proving Theorem 1.5:

Lemma 4.7. An ideal non-packing clutter does not identify to a minimally non-ideal clutter with covering number 2.

Proof. We make the following two claims:

Claim 1. For $n \geq 4$, every clutter that identifies to $\Delta_{n}$ has a delta minor and is therefore non-ideal.

Proof of Claim. Recall that $\Delta_{n}=\{\{1,2\},\{1,3\}, \ldots,\{1, n\},\{2,3, \ldots, n\}\}$. Assume that $\mathcal{C}$, a clutter over ground set $[n+1]$, identifies to $\Delta_{n}$. We may assume that the two coexclusive elements of $\mathcal{C}$ are either $1, n+1$ or $n, n+1$. In the first case, $b(\mathcal{C})$ has $\{2,3, \ldots, n\}$ as a member, and since $n \geq 4$, it also has two members among $\{1,2\},\{1,3\}, \ldots,\{1, n\}$ or two members among $\{n+1,2\},\{n+1,3\}, \ldots,\{n+1, n\}$. Either way, $\{2,3, \ldots, n\}$ is a doubly redundant member of $b(\mathcal{C})$, so by Theorem $2.1, b(\mathcal{C})$ and therefore $\mathcal{C}$ has a delta minor. In the second case when $n, n+1$ are the coexclusive elements of $\mathcal{C}, b(\mathcal{C})$ has $\{1,2\},\{1,3\}$ as members as well as

$$
\{2,3, \ldots, n-1, n\} \quad \text { or } \quad\{2,3, \ldots, n-1, n+1\}
$$


as a member. But again, both members above are doubly 1-redundant, so by Theorem $2.1, b(\mathcal{C})$ and therefore $\mathcal{C}$ has a delta minor, finishing the proof of the claim.

Claim 2. Take a clutter $\mathcal{C}$ over ground set $[2 n]$, for some $n \geq 2$. Assume that $1,2 n$ are coexclusive elements, and that $\left.\mathcal{C}\right|_{1=2 n}$ includes $\mathcal{C}_{2 n-1}^{2}$ as minimal covers. If $\mathcal{C}$ is ideal, then it packs.

Proof of Claim. Assume that $\mathcal{C}$, and therefore $b(\mathcal{C})$, is ideal. Since $\tau(\mathcal{C})=\tau\left(\left.\mathcal{C}\right|_{1=2 n}\right)=2$, it suffices to present two disjoint members of $\mathcal{C}$. Notice that $b(\mathcal{C})$ has

$$
\{2,3\},\{3,4\}, \ldots,\{2 n-2,2 n-1\}
$$

as members. If $b(\mathcal{C})$ had $\{1,2\},\{2 n-1,1\}$ as members also, then $\left(\frac{1}{2} \frac{1}{2} \cdots \frac{1}{2}\right)$ would be an extreme point of $Q(b(\mathcal{C}) \backslash 2 n)$, implying that $b(\mathcal{C}) \backslash 2 n$, and therefore its major $b(\mathcal{C})$, is non-ideal, which is not the case. By symmetry between 1 and $2 n$, we may therefore assume that $b(\mathcal{C})$ has

$$
\{1,2\},\{2 n-1,2 n\}
$$

as members. Since $\mathcal{C}$ is ideal, it has a fractional packing $y \in \mathbb{R}_{+}^{\mathcal{C}}$ of value 2. By Complementary Slackness, whenever $y_{C}>0, C$ must intersect every minimum cover exactly once. However, the only such element subsets are $\{1,3, \ldots, 2 n-1\}$ and $\{2,4, \ldots, 2 n\}$, and since the fractional packing has value 2 , these two subsets must be members of $\mathcal{C}$, which happen to be disjoint, thereby finishing the proof.

These claims, along with Theorem 4.6, finish the proof of the lemma.

We are now ready to prove Theorem 1.5, stating that if $\mathcal{C}$ is an ideal mnp clutter with coexclusive elements $e, f$, then either (i) $\left.\mathcal{C}\right|_{e=f}$ is ideal mnp, or (ii) $\left.\mathcal{C}\right|_{e=f}$ is not mnp and every mnp minor has covering number 2.

Proof of Theorem 1.5. If $\left.\mathcal{C}\right|_{e=f}$ is not mnp, then Corollary 4.3 implies that every mnp minor of it has covering number 2, so (ii) holds. We may therefore assume that $\left.\mathcal{C}\right|_{e=f}$ is mnp. To prove (i) holds, we need to show that $\left.\mathcal{C}\right|_{e=f}$ is ideal. Suppose otherwise. Then by Theorem $1.1,\left.\mathcal{C}\right|_{e=f}$ must be mni. Since $\mathcal{C}$ itself is ideal, Corollary 4.5 implies that $\left.\mathcal{C}\right|_{e=f}$ has covering number 2 . So we have an ideal non-packing clutter $\mathcal{C}$ identifying to an mni clutter with covering number 2 , contradicting Lemma 4.7. Thus, $\left.\mathcal{C}\right|_{e=f}$ is an ideal mnp clutter, showing that (i) holds.

\section{Ideal minimally non-packing clutters with covering number two}

In this section, we provide proofs of Theorem 1.6 and Proposition 1.7. 


\subsection{Proof of Theorem 1.6}

Let $\mathcal{C}$ be an ideal mnp clutter without coexclusive elements, where $\tau(\mathcal{C})=2$. Since $\mathcal{C}$ is mnp,

every element $e$ is contained in a minimum cover.

For if not, then $\tau(\mathcal{C} \backslash e)=\tau(\mathcal{C})$, and because $\mathcal{C}$ does not pack, $\mathcal{C} \backslash e$ does not pack either, contradicting the minimality of $\mathcal{C}$. In fact,

Claim 1. Every element is contained in exactly one minimum cover, i.e. the minimum covers partition the ground set, so (i) holds in Theorem 1.6.

Proof of Claim. Suppose for a contradiction that element $e$ is contained in minimum covers $\{e, f\},\{e, g\}$. Since $\mathcal{C}$ is ideal, it does not have a delta minor, so by Corollary 2.2, elements $f, g$ are coexclusive, a contradiction to our hypothesis.

Let's relabel the elements so that $E(\mathcal{C})=[2 n]$ and the minimum covers are $\{1,2\},\{3,4\}, \ldots,\{2 n-1,2 n\}$, for some integer $n \geq 1$. Since there is no ideal mnp clutter with less than 6 elements, it follows that $n \geq 3$. Notice that each member has size at least $n$, as it contains an element from each minimum cover. In fact,

Claim 2. The minimum size of a member is $n=\frac{|E(\mathcal{C})|}{2}$, so (ii) holds in Theorem 1.6.

Proof of Claim. It suffices to present a member of size $n$. Well, since $\mathcal{C}$ is ideal, it has a fractional packing $y \in \mathbb{R}_{+}^{\mathcal{C}}$ of value 2 . Pick a member $C$ for which $y_{C}>0$. By Complementary Slackness, $C$ must intersect every minimum cover exactly once, i.e. $|C|=n$.

Now let $\mathcal{C}_{0}:=\{C \in \mathcal{C}:|C|=n\}$. As argued above, every fractional packing of value 2 only picks members from $\mathcal{C}_{0}$, so $\tau\left(\mathcal{C}_{0}\right) \geq 2$, and since $2=\tau(\mathcal{C}) \geq \tau\left(\mathcal{C}_{0}\right)$, we get that $\tau\left(\mathcal{C}_{0}\right)=2$. (In fact, by Strict Complementarity, $\mathcal{C}_{0}$ consists precisely of members that are used in at least one fractional packing of value 2.) Moreover, as $\mathcal{C}$ does not have two disjoint members, $\mathcal{C}_{0}$ has no two disjoint members, so $\mathcal{C}_{0}$ does not pack. In fact,

Claim 3. $\mathcal{C}_{0}$ is an ideal non-packing clutter with $\tau\left(\mathcal{C}_{0}\right)=2$, so (iii) holds in Theorem 1.6.

Proof of Claim. We just showed above that this clutter has covering number 2 and is non-packing. It remains to show that $\mathcal{C}_{0}$ is ideal. We will use Lemma 3.1. By this tool, it suffices to show that

$$
\operatorname{conv}\left\{\chi_{C}: C \in \mathcal{C}_{0}\right\} \supseteq Q\left(b\left(\mathcal{C}_{0}\right)\right) \cap\left\{x: x_{2 i-1}+x_{2 i}=1 \forall i \in[n]\right\} .
$$

To this end, pick a point $x^{\star}$ in the set on the right-hand-side. As $Q\left(b\left(\mathcal{C}_{0}\right)\right) \subseteq Q(b(\mathcal{C}))$, we have $x^{\star} \in Q(b(\mathcal{C}))$. Since $\mathcal{C}$ is ideal, so is $b(\mathcal{C})$, implying that for some $\lambda \in \mathbb{R}_{+}^{\mathcal{C}}$ with $\sum_{C \in \mathcal{C}} \lambda_{C}=1$, we have that

$$
x^{\star} \geq \sum_{C \in \mathcal{C}} \lambda_{C} \chi_{C}
$$

Since for each $i \in[n], x_{2 i-1}^{\star}+x_{2 i}^{\star}=1$ and $\{2 i-1,2 i\}$ is a cover of $\mathcal{C}$, equality must hold above and, for all $C \in \mathcal{C}-\mathcal{C}_{0}, \lambda_{C}=0$. Hence, $x^{\star} \in \operatorname{conv}\left\{\chi_{C}: C \in \mathcal{C}_{0}\right\}$, proving the claim. 
Finally, we will show that $\mathcal{C}_{0}$ is a marginal cuboid. To prove this, we need to show $\mathcal{C}_{0}$ is the cuboid of a set of $n$-dimensional points satisfying (C1), (C2) and (C3). Consider the map

$$
\varphi:\left\{x \in\{0,1\}^{2 n}: x_{2 i-1}+x_{2 i}=1 \forall i \in[n]\right\} \rightarrow\{0,1\}^{n}
$$

where $\varphi(x):=\left(x_{1}, x_{3}, \ldots, x_{2 n-1}\right)$. Let $S:=\left\{\varphi\left(\chi_{C}\right): C \in \mathcal{C}_{0}\right\}$.

Claim 4. $S \subseteq\{0,1\}^{n}$ satisfies

(C1) for each $i \in[n],\left\{x \in S: x_{i}=0\right\} \neq \emptyset$ and $\left\{x \in S: x_{i}=1\right\} \neq \emptyset$,

(C2) if $x \in S$ then $\mathbf{1}-x \notin S$, and

(C3) $\operatorname{conv}(S)=\left\{y \in[0,1]^{n}: \sum\left(y_{i}: 2 i-1 \in B\right)+\sum\left(1-y_{j}: 2 j \in B\right) \geq 1 \quad \forall B \in b\left(\mathcal{C}_{0}\right)\right\}$.

In particular, $\mathcal{C}_{0}$ is a marginal cuboid, so (iv) holds in Theorem 1.6.

Proof of Claim. (C1) holds because $\tau\left(\mathcal{C}_{0}\right) \neq 1$, and (C2) holds because $\mathcal{C}_{0}$ does not have two disjoint members. It remains to prove $(\mathrm{C} 3)$ - call the right side of the equation $Y .(\subseteq)$ Let $C \in \mathcal{C}_{0}$ and $y^{\star}:=\varphi\left(\chi_{C}\right)$. It suffices to show that $y^{\star} \in Y$. To this end, take $B \in b\left(\mathcal{C}_{0}\right)$. Then

$$
\begin{aligned}
\sum\left(y_{i}^{\star}: 2 i-1 \in B\right)+\sum\left(1-y_{j}^{\star}: 2 j \in B\right) & =\sum(1: 2 i-1 \in C \cap B)+\sum(1: 2 j \in C \cap B) \\
& =|C \cap B| \\
& \geq 1 .
\end{aligned}
$$

Since this is true for all such $B$ 's, it follows that $y^{\star} \in Y .(\supseteq)$ Conversely, take a point $y^{\star} \in Y$. Let $x^{\star}:=$ $\left(y_{1}^{\star}, 1-y_{1}^{\star}, y_{2}^{\star}, 1-y_{2}^{\star}, \ldots, y_{n}^{\star}, 1-y_{n}^{\star}\right)$. We claim that $x^{\star} \in Q\left(b\left(\mathcal{C}_{0}\right)\right)$. To this end, take $B \in b\left(\mathcal{C}_{0}\right)$. Then

$$
\begin{aligned}
x^{\star}(B) & =\sum\left(x_{2 i-1}^{\star}: 2 i-1 \in B\right)+\sum\left(x_{2 j}^{\star}: 2 j \in B\right) \\
& =\sum\left(y_{i}^{\star}: 2 i-1 \in B\right)+\sum\left(1-y_{j}^{\star}: 2 j \in B\right) \\
& \geq 1 .
\end{aligned}
$$

Since this is true for all such $B$ 's, it follows that $x^{\star} \in Q\left(b\left(\mathcal{C}_{0}\right)\right)$. However, we know by Claim 3 that $\mathcal{C}_{0}$, and therefore $b\left(\mathcal{C}_{0}\right)$, is ideal. And because for each $i \in[n], x_{2 i-1}^{\star}+x_{2 i}^{\star}=1$, it must be that $x^{\star} \in \operatorname{conv}\left\{\chi_{C}: C \in\right.$ $\left.\mathcal{C}_{0}\right\}$, implying in turn that $y^{\star} \in \operatorname{conv}\left\{\varphi\left(\chi_{C}\right): C \in \mathcal{C}_{0}\right\}=\operatorname{conv}(S)$, proving (C3).

Hence, every ideal mnp clutter, without coexclusive elements, of covering number 2 satisfies (i)-(iv), proving Theorem 1.6.

\subsection{The $Q_{r, t}$ 's are marginal cuboids without coexclusive elements.}

For an integer $n \geq 1$, let $H_{n}$ denote the $\left(2^{n}-1\right) \times n$ matrix whose columns are indexed by $[n]$ and whose rows are the incidence vectors of the nonempty subsets of $[n]$. Given that $J$ is the all-ones matrix of the same 
dimensions, let $H_{n}^{\star}$ denote the matrix satisfying $H_{n}+H_{n}^{\star}=J$. For integers $r, t \geq 1$, Cornuéjols et al. [5] denote by $Q_{r, t}$ the clutter over $2(r+t+1)$ many elements whose incidence matrix is

$$
M\left(Q_{r, t}\right)=\left(\begin{array}{cccccc}
H_{r} & H_{r}^{\star} & J & \mathbf{0} & 1 & 0 \\
H_{r}^{\star} & H_{r} & \mathbf{0} & J & 1 & 0 \\
J & \mathbf{0} & H_{t}^{\star} & H_{t} & 0 & 1 \\
\mathbf{0} & J & H_{t} & H_{t}^{\star} & 0 & 1
\end{array}\right) .
$$

It is worth mentioning that $Q_{1,1}$ is in fact $Q_{6}$. Notice that $\tau\left(Q_{r, t}\right)=2$ and that the minimum covers partition the ground set. A simple argument shows that all proper minors of $Q_{r, t}$ pack, and since $Q_{r, t}$ itself does not pack, we get that $Q_{r, t}$ is mnp. Theorem 1.1 now implies that either $Q_{r, t}$ is ideal or mni. And it follows from Theorem 4.6 that $Q_{r, t}$ cannot be mni, so $Q_{r, t}$ is an ideal mnp clutter.

Remark 5.1. For integers $r, t \geq 1$, the clutter $Q_{r, t}$ does not have coexclusive elements.

Proof. Label the columns of $M\left(Q_{r, t}\right)$ by $e_{1}, \ldots, e_{r}, f_{1}, \ldots, f_{r}, e_{1}^{\prime}, \ldots, e_{t}^{\prime}, f_{1}^{\prime}, \ldots, f_{t}^{\prime}, e, f$ in this order. The minimal covers

- $\{e, f\},\left\{e_{i}, f_{i}\right\},\left\{e_{j}^{\prime}, f_{j}^{\prime}\right\}$ for $i \in[r]$ and $j \in[t]$,

- $\left\{e_{i}, f_{j}, e_{i^{\prime}}^{\prime}, f_{j^{\prime}}^{\prime}\right\}$ for $i, j \in[r]$ and $i^{\prime}, j^{\prime} \in[t]$ where $i \neq j$ and $i^{\prime} \neq j^{\prime}$,

- $\left\{e_{1}, \ldots, e_{r}, f_{i}^{\prime}, f\right\},\left\{f_{1}, \ldots, f_{r}, e_{i}^{\prime}, f\right\}$ for $i \in[t]$, and

- $\left\{e_{1}^{\prime}, \ldots, e_{t}^{\prime}, e_{i}, e\right\},\left\{f_{1}^{\prime}, \ldots, f_{t}^{\prime}, f_{i}, e\right\}$ for $i \in[r]$,

show that every pair of elements are used in a minimal cover and therefore are not coexclusive.

Thus, we can apply Theorem 1.6 to each $Q_{r, t}$. Notice that each member has size $r+t+1=\frac{\left|E\left(Q_{r, t}\right)\right|}{2}$, so (iv) implies that $Q_{r, t}$ itself is a marginal cuboid, and it is the cuboid of the rows of

$$
\left(\begin{array}{ccc}
H_{r} & J & 1 \\
H_{r}^{\star} & \mathbf{0} & 1 \\
J & H_{t}^{\star} & 0 \\
\mathbf{0} & H_{t} & 0
\end{array}\right)
$$

The $Q_{r, t}$ 's are not the only marginal cuboids that are mnp and without coexclusive elements $-Q_{10}$ proves this. As another example, the 10-dimensional marginal cuboid of

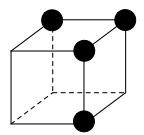

00

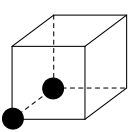

10

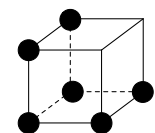

01

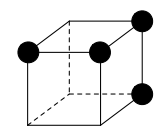

11

is also mnp and without coexclusive elements, and it is different from the $Q_{r, t}$ 's. 


\subsection{Marginal cuboids: Proposition 1.7}

We just proved Theorem 1.6 which, at its core, shows that the minimum size members of an ideal mnp clutter with covering number 2 , is another ideal non-packing clutter with the same covering number. The result also makes an attempt to explain this curiosity by showing that these members form a marginal cuboid. Here we complement this attempt by proving that marginal cuboids are always ideal non-packing clutters with covering number 2:

Proof of Proposition 1.7. Take an integer $n \geq 3$, and a set $S \subseteq\{0,1\}^{n}$ where (C1) for each $i \in[n],\{y \in S$ : $\left.y_{i}=0\right\} \neq \emptyset$ and $\left\{y \in S: y_{i}=1\right\} \neq \emptyset$, (C2) whenever $y \in S$ then $\mathbf{1}-y \notin S$, and (C3) the convex hull of $S$ can be described with hypercube and generalized set covering inequalities. Let $\mathcal{C}$ be the cuboid of $S$. It is clear that $\tau(\mathcal{C})=2$ by $(\mathrm{C} 1)$, and that $\mathcal{C}$ has no disjoint members by $(\mathrm{C} 2)$. It remains to show that $\mathcal{C}$ is an ideal clutter. Well, (C3) states that

$$
\operatorname{conv}\{y: y \in S\}=\left\{y \in[0,1]^{n}: \sum\left(y_{i}: i \in I\right)+\sum\left(1-y_{j}: j \in J\right) \geq 1 \quad \forall(I, J) \in \mathcal{V}\right\}
$$

for some appropriate set $\mathcal{V}$. We may assume that for each $(I, J) \in \mathcal{V}, I \cap J=\emptyset$. After a change of variables, we see that the above equation implies the following:

$$
\operatorname{conv}\left\{\chi_{C}: C \in \mathcal{C}\right\}=\left\{x \in \mathbb{R}_{+}^{2 n}: \begin{array}{l}
\sum\left(x_{2 i-1}: i \in I\right)+\sum\left(x_{2 j}: j \in J\right) \geq 1 \quad \forall(I, J) \in \mathcal{V} \\
x_{2 i-1}+x_{2 i}=1 \quad \forall i \in[n]
\end{array}\right\} .
$$

Together with Lemma 3.1, this equation implies that $\mathcal{C}$ is an ideal clutter, as required.

\section{Bounded degree cuboids}

Here we prove Theorems 1.9 and 1.10. Take an integer $n \geq 1$ and a subset $S \subseteq\{0,1\}^{n}$. Fix an index $i \in[n]$. After dropping the $i^{\text {th }}$ coordinate from every point of $S \cap\left\{x: x_{i}=0\right\}$, we obtain a subset of $\{0,1\}^{n-1}$ that we refer to as the 0-restriction of $S$ over $i$. The 1-restriction of $S$ over $i$ is defined similarly. The projection of $S$ over $i$ is the subset of $\{0,1\}^{n-1}$ obtained after dropping the $i^{\text {th }}$ coordinate from each point of $S$. For instance, over index 3 , the 0 -restriction of $\{100,010,001,111\}$ is $\{10,01\}$, its 1 -restriction is $\{00,11\}$ and its projection is $\{10,01,00,11\}$.

Remark 6.1. Take $n \geq 1, S \subseteq\{0,1\}^{n}$ and its cuboid $\mathcal{C}$. Then, for each $i \in[n]$, the following statements hold:

(i) $\mathcal{C} \backslash(2 i-1) / 2 i$ is the cuboid of the 0 -restriction of $S$ over $i$,

(ii) $\mathcal{C} /(2 i-1) \backslash 2 i$ is the cuboid of the 1 -restriction of $S$ over $i$,

(iii) $\mathcal{C} /\{2 i-1,2 i\}$ is the cuboid of the projection of $S$ over $i$.

Justified by this remark, if $S^{\prime}$ is obtained from $S$ after a series of 0-restrictions, 1-restrictions and projections, we say that the cuboid of $S^{\prime}$ is a cuboid minor of the cuboid of $S$. Take an integer $k \geq 1$. Recall that the cuboid 
of $S$ has degree at most $k$ if $G_{n}\left[\{0,1\}^{n}-S\right]$ has maximum degree at most $k$, and the cuboid of $S$ has degree $k$ if it has degree at most $k$ and not $k-1$. It can be readily checked that if a cuboid has degree at most $k$, then so does every cuboid minor of it.

In $\S 6.1$, we prove Theorem 1.9 stating that every non-packing cuboid of degree at most 2 has one of $Q_{6}, Q_{2,1}, Q_{10}$ as a cuboid minor, whose incidence matrices are displayed below. Even though these minors are quite large, we will find them without much difficulty by exploiting the geometric interpretation of minor operations provided in Remark 6.1.

$$
\begin{aligned}
& M\left(Q_{6}\right)=\left(\begin{array}{llllll}
1 & & 1 & & 1 & \\
1 & & & 1 & & 1 \\
& 1 & 1 & & & 1 \\
& 1 & & 1 & 1 &
\end{array}\right)
\end{aligned}
$$

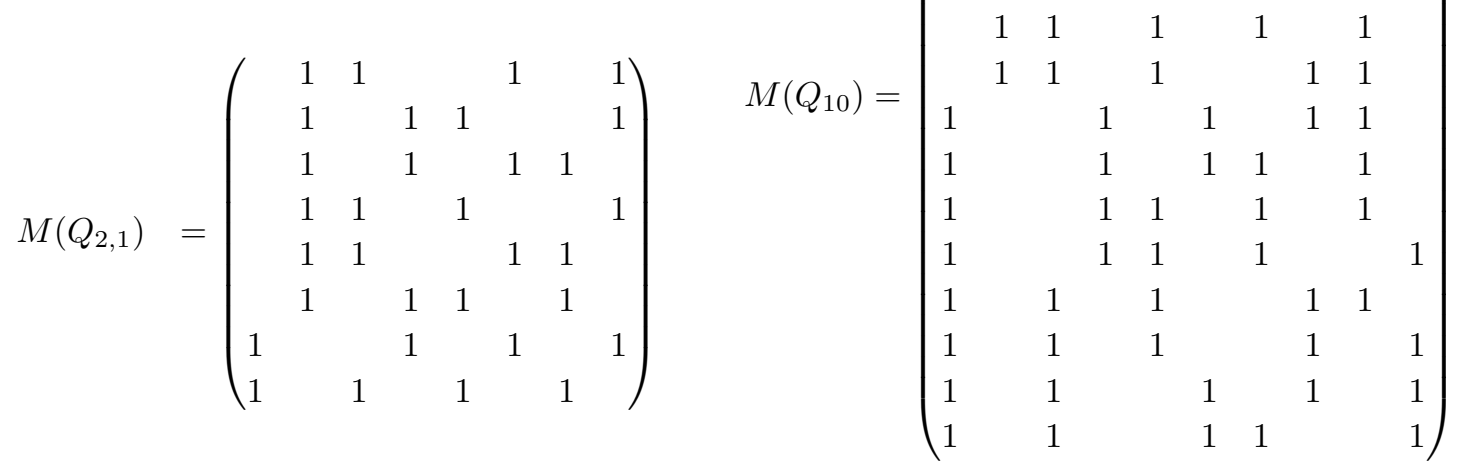

We will then move on to non-packing cuboids of bounded degree. In $\S 6.2$, we prove Theorem 1.10 (i), stating that a non-packing cuboid of bounded degree and large dimension has one of $Q_{3}, Q_{6}$ as a cuboid minor. A common ingredient for the proofs of Theorems 1.9 and 1.10 (i) is the following:

Lemma 6.2. Take integers $n, k \geq 1$ and a subset $S \subseteq\{0,1\}^{n}$ whose cuboid is non-packing of degree at most $k$. If $n \geq 2 k+1$, then $|S|=2^{n-1}$ and so $G_{n}[S] \cong G_{n}\left[\{0,1\}^{n}-S\right]$.

Proof. Suppose that $n \geq 2 k+1$. As the cuboid of $S$ is non-packing, we have that (C2) if $x \in S$, then $\mathbf{1}-x \notin S$. In particular, $|S| \leq 2^{n-1}$. Suppose for a contradiction that equality does not hold. Then $\{0,1\}^{n}-S$ must contain antipodal points, say $y$ and $\mathbf{1}-y$. Since $\mathbf{1}-y$ has degree at most $k$ in $G_{n}\left[\{0,1\}^{n}-S\right]$, it follows from (C2) that $y$ has at most $k$ neighbors in $G_{n}[S]$. However, $y$ itself has degree at most $k$ in $G_{n}\left[\{0,1\}^{n}-S\right]$, implying that $y$ has at most $k+k=2 k$ neighbors in total. Since $y$ has exactly $n$ neighbors in $G_{n}$, we get that $n \leq 2 k$, a contradiction. As a result, $x \in S$ if and only if $\mathbf{1}-x \notin S$, implying in turn that $G_{n}[S] \cong G_{n}\left[\{0,1\}^{n}-S\right]$.

We will then use Theorem 1.10 (i) to prove Theorem 1.10 (ii) in $\S 6.3$. As pointed out earlier, a caveat of bounded degree cuboids is that they are not necessarily ideal. However, proved in $\S 6.4$, Theorem 1.10 (iii) says that these cuboids can be made ideal after excluding only finitely many non-ideal minors. 


\subsection{Proof of Theorem 1.9}

Take an integer $n \geq 1$ and a set $S \subseteq\{0,1\}^{n}$ where

(C1) for each $i \in[n],\left\{x \in S: x_{i}=0\right\} \neq \emptyset$ and $\left\{x \in S: x_{i}=1\right\} \neq \emptyset$,

(C2) if $x \in S$ then $\mathbf{1}-x \notin S$, and

(C3') $G_{n}\left[\{0,1\}^{n}-S\right]$ has maximum degree at most two.

Notice that (C1) and (C2) imply that $n \geq 3$. Let $\mathcal{C}$ be the cuboid of $S$. We will show that $\mathcal{C}$ has one of $Q_{6}, Q_{2,1}, Q_{10}$ as a cuboid minor, thereby proving Theorem 1.9. (In fact, we will obtain one of $Q_{6}, Q_{2,1}, Q_{10}$ after applying a series of 0 - and 1-restrictions.)

Claim 1. $G_{n}[S]$ also has maximum degree at most two.

Proof of Claim. Observe that the vertex-induced subgraph $G_{n}[S]$ is isomorphic to $G_{n}[\mathbf{1}-x: x \in S]$, and since (C2) holds, $G_{n}[\mathbf{1}-x: x \in S]$ is a subgraph of $G_{n}\left[\{0,1\}^{n}-S\right]$, which by (C3') has maximum degree at most two. Thus, $G_{n}[S]$ also has maximum degree at most two.

Notice that (C2) implies that $|S| \leq 2^{n-1}$.

Claim 2. If $n=3$ then $\mathcal{C}$ is $Q_{6}$.

Proof of Claim. Note that $4=2^{2} \geq|S|$. It follows from (C1) and (C2) that $|S| \geq 3$. Moreover, since $G_{3}\left[\{0,1\}^{3}-S\right]$ has maximum degree at most two, it follows that $|S|=4$. As $G_{n}[S]$ also has maximum degree at most two by Claim $1, G_{3}[S]$ must consist of 4 isolated vertices, implying that $\mathcal{C}$ is $Q_{6}$.

We may therefore assume that $n \geq 4$.

Claim 3. If $|S|<2^{n-1}$, then $n=4$ and there is a subset $S^{\prime} \subseteq\{0,1\}^{4}$ containing $S$ where $\left|S^{\prime}\right|=8$, $S^{\prime}$ does not contain antipodal points, and $G_{4}\left[S^{\prime}\right]$ has maximum degree at most two.

Proof of Claim. Assume that $|S|<2^{n-1}$. It follows from Lemma 6.2 that $n \leq 2 \cdot 2=4$, so $n=4$. From each antipodal pair that has an empty intersection with $S$ pick a point, and label the chosen points as $x^{1}, \ldots, x^{k}$. Let $S^{\prime}:=S \cup\left\{x^{1}, \ldots, x^{k}\right\}$. It is clear that $\left|S^{\prime}\right|=8$ and $S^{\prime}$ does not contain antipodal points. Note that $G_{4}\left[S^{\prime}\right]$ is isomorphic to $G_{4}\left[\{0,1\}^{4}-S^{\prime}\right]$, which is a subgraph of $G_{4}\left[\{0,1\}^{4}-S\right]$, implying in turn that $G_{4}\left[S^{\prime}\right]$ has maximum degree at most two, as claimed.

By the end of Claim 5, we will have shown that $S^{\prime}=S$. But for now, temporarily set

$$
R:= \begin{cases}S^{\prime} & \text { if } n=4 \\ S & \text { if } n \geq 5\end{cases}
$$

Since $R$ picks exactly one point from each antipodal pair, it follows that $G_{n}[R]$ and $G_{n}\left[\{0,1\}^{n}-R\right]$ are isomorphic and have maximum degree at most two. A subcube of $\{0,1\}^{n}$ is what is obtained after fixing $n-3$ many of the variables. 
Claim 4. The subgraph $G_{n}[R]$ restricted to every subcube is one of the nine uncoordinated configurations displayed in Figure 2.

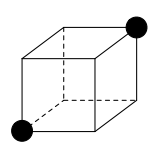

1

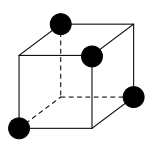

5

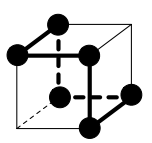

2

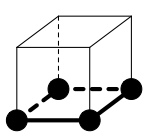

6

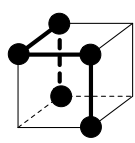

3

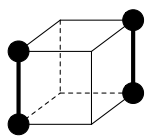

7

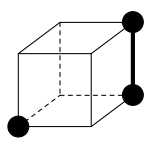

4

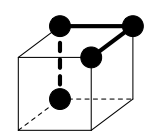

8

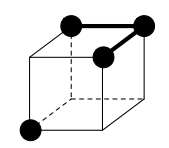

9

Figure 2: Possible configurations of each subcube. Bold vertices are the points in $R$.

Proof of Claim. Take an arbitrary subcube and denote by $H$ its skeleton graph which has 8 vertices. Since $H[R], H\left[\{0,1\}^{n}-R\right]$ are respectively subgraphs of $G_{n}[R], G_{n}\left[\{0,1\}^{n}-R\right]$ that have maximum degree at most two, $H[R]$ and $H\left[\{0,1\}^{n}-R\right]$ also have maximum degree at most two. This implies that $R$ picks at least 2 and at most 6 points of the subcube. If $R$ picks exactly 2 (resp. 6) points, then it is clear that the subcube has configuration (1) (resp. (2)). If $R$ picks 5 (resp. 3) points, then it is clear that the subcube obeys configuration (3) (resp. (4)). Finally, when $R$ picks 4 points of the subcube, a simple enumeration shows that the subcube takes one of (5)-(9) as disguise.

Claim 5. If $n=4$, then either $\mathcal{C}$ is $Q_{2,1}$ or $\mathcal{C}$ has a $Q_{6}$ cuboid minor.

Proof of Claim. Suppose $n=4$. Let $H_{0}$ (resp. $H_{1}$ ) be the skeleton of the subcube obtained after fixing $x_{4}=$ 0 (resp. $x_{4}=1$ ). By Claim $4, H_{0}\left[S^{\prime}\right]$ and $H_{1}\left[S^{\prime}\right]$ each obey one of the nine configurations displayed in Figure 2. Since $S^{\prime}$ picks exactly one point from each antipodal pair, the configuration of $H_{0}\left[S^{\prime}\right]$ determines the configuration of $H_{1}\left[S^{\prime}\right]$. So because $G_{4}\left[S^{\prime}\right]$ and $G_{4}\left[\{0,1\}^{4}-S^{\prime}\right]$ have maximum degree at most two, configurations (3), (4), (6), (8) are not plausible. Hence, $H_{0}\left[S^{\prime}\right]$ and $H_{1}\left[S^{\prime}\right]$ are of the form (1), (2), (5), (7), or (9). It is now easy to see that if $S \subsetneq S^{\prime}$, then $G_{4}\left[\{0,1\}^{4}-S\right]$ has a vertex of degree more than two. This implies that $S^{\prime}=S$.

If $H_{0}[S]$ takes one of configurations (1), (2), (9) as disguise, then $\mathcal{C}$ is $Q_{2,1}$. If $H_{0}[S]$ takes (5) as disguise, then $\mathcal{C}$ has a $Q_{6}$ cuboid minor. We may therefore assume that $H_{0}[S]$ takes (7) as disguise, which subsequently implies that $H_{1}[S]$ also takes (7) as disguise, as shown below: 


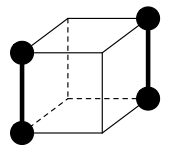

$H_{0}[S]$

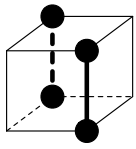

$H_{1}[S]$

By 0-restricting the vertical dimension, we see that $\mathcal{C}$ has a $Q_{6}$ cuboid minor, as claimed.

We may therefore assume that $n \geq 5$. After this point, we will fix the variables $x_{6}, \ldots, x_{n}$ to 0 , and focus on the 5 -dimensional hypercube induced on variables $x_{1}, \ldots, x_{5}$ (where properties $(\mathrm{C} 1)-(\mathrm{C} 2)$ are no longer applicable). Let $H$ be the skeleton of this hypercube, and for $i, j \in\{0,1\}$, let $H_{i j}$ be the skeleton of the subcube obtained after fixing $x_{4}=i, x_{5}=j$ :

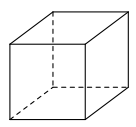

$H_{00}$

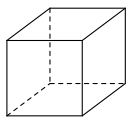

$H_{10}$

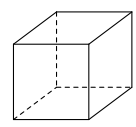

$H_{01}$

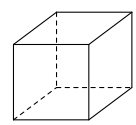

$H_{11}$

Let $T \subseteq\{0,1\}^{5}$ be obtained from $\left\{x \in S: x_{6}=\cdots=x_{n}=0\right\}$ after dropping variables $x_{6}, \ldots, x_{n}$. Let $\mathcal{C}^{\prime}$ be the cuboid of $T$ - note that $\mathcal{C}^{\prime}$ is a cuboid minor of $\mathcal{C}$. Note that $H[T], H\left[\{0,1\}^{5}-T\right]$ are (isomorphic to) subgraphs of $G_{n}[S], G_{n}\left[\{0,1\}^{n}-S\right]$, respectively, and therefore have maximum degree at most two. Note further that the subgraph $H[T]$ restricted to each subcube takes one of the configurations (1)-(9) as disguise. We will use these facts to show that $\mathcal{C}^{\prime}$ has one of $Q_{6}, Q_{2,1}, Q_{10}$ as a cuboid minor. ${ }^{5}$ We will also be following the coordinate system shown below on each subcube:

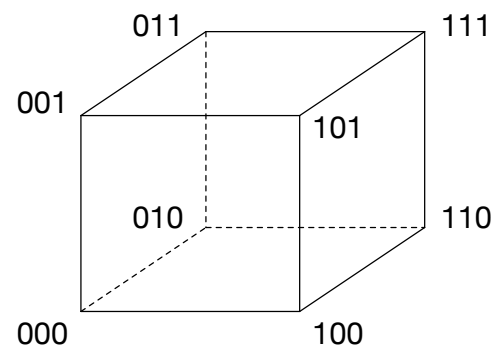

Claim 6. The following statements hold:

(i) if a subcube of $H[T]$ is of the form (1) or (2), then $\mathcal{C}^{\prime}$ has a $Q_{2,1}$ cuboid minor,

(ii) if a subcube of $H[T]$ is of the form (3) or (4), then $\mathcal{C}^{\prime}$ has a $Q_{6}$ cuboid minor,

${ }^{5} \mathrm{~A}$ cuboid minor of $\mathcal{C}^{\prime}$ is also a cuboid minor of $\mathcal{C}$. 
(iii) if a subcube of $H[T]$ is of the form (5), then $\mathcal{C}^{\prime}$ has a $Q_{6}$ cuboid minor,

(iv) if a subcube of $H[T]$ is of the form (6), then $\mathcal{C}^{\prime}$ has a $Q_{6}$ cuboid minor.

Proof of Claim. By symmetry, we may assume that the subcube in each of the four cases is $H_{00}$. (i) Since $H[T], H\left[\{0,1\}^{5}-T\right]$ have maximum degree at most two, it follows if $H_{00}$ is of the form (1) (resp. (2)), then $H_{01}$ is of the form (2) (resp. (1)):

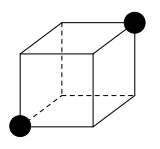

$H_{00}$

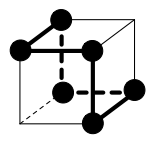

$H_{01}$

implying that $\mathcal{C}^{\prime}$ has a $Q_{2,1}$ cuboid minor, as required. (ii) Suppose $H_{00}$ takes on form (3):

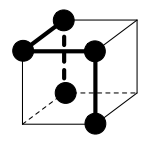

$H_{00}$

We will show that one of $H_{01}, H_{10}$ takes on form (4). It suffices to show that either $00001,11101,11001 \in T$ or $00010,11110,11010 \in T$. Since $H[T]$ has maximum degree at most two, it follows that $00101,10101,01101 \notin$ $T$. Thus, as $H\left[\{0,1\}^{5}-T\right]$ also has maximum degree at most two, it must be that $00001,11101 \in T$. Similarly, $00010,11110 \in T$. The point $11000 \notin T$ is adjacent to $11100 \notin T$, so one of its neighbors 11001,11010 must belong to $T$, implying in turn that either $H_{01}$ or $H_{10}$ is of the form (4). By symmetry, we may assume that $H_{01}$ is of the form (4):

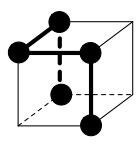

$H_{00}$

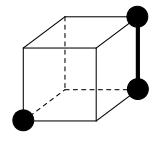

$H_{01}$

After setting $x_{3}=x_{4}=0$, it is easy to see that $\mathcal{C}^{\prime}$ has a $Q_{6}$ cuboid minor, as required. By the symmetry between the points in $T$ and the points outside $T$, if $H_{00}$ takes on form (4), then $\mathcal{C}^{\prime}$ has a $Q_{6}$ cuboid minor as well. (iii) is immediate. (iv) Suppose $H_{00}$ takes on form (6). The degree conditions on $H[T]$ and $H\left[\{0,1\}^{5}-T\right]$ imply that $H[T]$ looks as follows: 


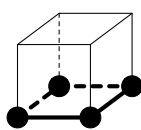

$H_{00}$

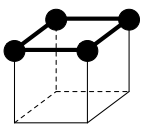

$H_{10}$

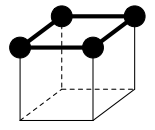

$H_{01}$

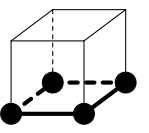

$H_{11}$

After setting $x_{1}=x_{2}=0$, we see that $\mathcal{C}^{\prime}$ has a $Q_{6}$ cuboid minor, as claimed.

Claim 7. If a subcube of $H[T]$ is of the form (8), then either $\mathcal{C}^{\prime}$ is $Q_{10}$ or $\mathcal{C}^{\prime}$ has a $Q_{6}$ cuboid minor.

Proof of Claim. We may assume that $H_{00}$ is of the form (8). It is easy to see that $01101,11101,01110,11110 \notin$ $T$ and $00001,10001,00010,10010 \in T$. In the first case, assume that $10110 \in T$. Then $11010,00110 \notin T$ and $01010 \in T$. These imply that $00011,10011 \notin T$ and $01111,11111 \in T$. So $00101,11001,00111,11011 \in T$ and $01001,10101,01011,10111 \notin T$ :

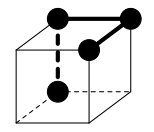

$H_{00}$

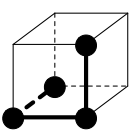

$H_{10}$

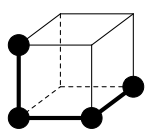

$H_{01}$

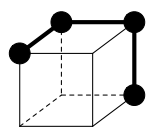

$H_{11}$

implying that $\mathcal{C}^{\prime}$ is $Q_{10}$. Otherwise, we have $10110 \notin T$. Similarly, we may assume that $01010,01001,10101 \notin$ $T$. Then $11010,00110,11001,00101 \in T$. These imply that $01111,11111 \in T$ and $00011,10011 \notin T$, so $01011,10111 \in T$ and $00111,11011 \notin T:$

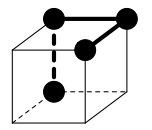

$H_{00}$

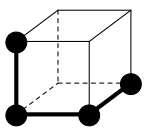

$H_{10}$

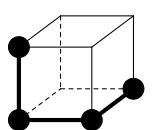

$H_{01}$

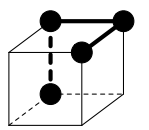

$H_{11}$

Setting $x_{1}=1, x_{2}=0$, we see that $\mathcal{C}^{\prime}$ has a $Q_{6}$ cuboid minor, finishing the proof.

We may therefore assume that every subcube of $H[T]$ is of the form (7) or (9). 
Claim 8. If a subcube of $H[T]$ is of the form (9), then $\mathcal{C}^{\prime}$ has a $Q_{6}$ cuboid minor.

Proof of Claim. We may assume that $H_{00}$ is of the form (9). Then $11101 \notin T$ and $11001 \in T$. Since every subcube of $H[T]$, including the one formed by the top two squares of $H_{00}$ and $H_{01}$, is of the form (7) or (9), it follows that $01101,10101 \notin T$ and $00101 \in T$, as well as $01001,10001 \in T$ and $00001 \notin T$ :
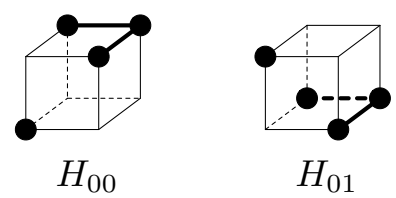

Setting $x_{1}=x_{4}=0$, we see that $\mathcal{C}^{\prime}$ has a $Q_{6}$ cuboid minor.

We may therefore assume that every subcube of $H[T]$ is of the form (7). Well, $H_{00}$ is of the form (7). Since every subcube of $H[T]$, including the one formed by the front two squares of $H_{00}$ and $H_{01}$, is of the form (7), it follows that $H_{01}$ is of the following form:
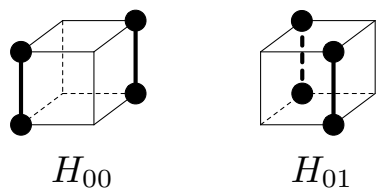

Setting $x_{3}=x_{4}=0$, we see that $\mathcal{C}^{\prime}$ has a $Q_{6}$ cuboid minor. This finishes the proof of Theorem 1.9.

\subsection{Proof of Theorem 1.10 (i)}

Take integers $n, k \geq 1$ and $S \subseteq\{0,1\}^{n}$ such that

(C1) for each $i \in[n],\left\{x \in S: x_{i}=0\right\} \neq \emptyset$ and $\left\{x \in S: x_{i}=1\right\} \neq \emptyset$,

(C2) if $x \in S$ then $\mathbf{1}-x \notin S$.

Assume further that $G_{n}\left[\{0,1\}^{n}-S\right]$ has maximum degree at most $k$, and $n \geq 4 k+2$. Note that (C1) and (C2) imply that $n \geq 3$. Let $\mathcal{C}$ be the cuboid of $S$. We will show that $\mathcal{C}$ has one of $Q_{3}, Q_{6}$ as a cuboid minor, thereby finishing the proof of Theorem 1.10 (i).

Since $n \geq 4 k+2 \geq 2 k+1$, Lemma 6.2 implies that $G_{n}[S] \cong G_{n}\left[\{0,1\}^{n}-S\right]$. In particular, $G_{n}[S]$ also has maximum degree at most $k$. Denote by 0 the zero point and by $e_{1}, \ldots, e_{n}$ the unit points in $\{0,1\}^{n}$. Without loss of generality, we have $\mathbf{0} \notin S$ and since $\mathbf{0}$ has degree at most $k$ in $G_{n}\left[\{0,1\}^{n}-S\right]$, we also have $e_{1}, \ldots, e_{n-k} \in S$. Let $X:=\left\{e_{i}+e_{j}: i, j \in[n-k], i \neq j\right\}$.

Claim 1. $\frac{k}{n-k-1} \cdot|X| \geq|S \cap X|$.

Proof of Claim. Let $a:=\left|\left\{\left(e_{i}, e_{i}+e_{j}\right): i \in[n-k], e_{i}+e_{j} \in S \cap X\right\}\right|$. On the one hand, each point in $X$ has exactly two neighbors among $e_{1}, \ldots, e_{n-k}$, so $a=2|S \cap X|$. On the other hand, as $G_{n}[S]$ has maximum degree $k$, each point among $e_{1}, \ldots, e_{n-k}$ has at most $k$ neighbors in $S \cap X$, so $(n-k) k \geq a$. Thus, $(n-k) k \geq 2|S \cap X|$, thereby implying the desired inequality. 
Claim 2. There exist distinct indices $p, q, r \in[n-k]$ such that $\left\{e_{p}+e_{q}, e_{q}+e_{r}, e_{r}+e_{p}\right\} \cap S=\emptyset$.

Proof of Claim. Suppose otherwise. Then for all distinct $p, q, r \in[n-k]$, at least one of $e_{p}+e_{q}, e_{q}+e_{r}, e_{r}+e_{p}$ is in $S$. As a result,

$$
|S \cap X| \geq \frac{\left(\begin{array}{c}
n-k \\
3
\end{array}\right)}{n-k-2}=\frac{1}{3}|X| .
$$

Together with Claim 1, this implies that $\frac{k}{n-k-1} \geq \frac{1}{3}$, so $4 k+1 \geq n$, a contradiction as $n \geq 4 k+2$.

We may assume that $(p, q, r)=(1,2,3)$. Consider now the subcube of $G_{n}[S]$ obtained after restricting $x_{4}=\cdots=x_{n}=0$. Depending on whether or not $e_{1}+e_{2}+e_{3} \in S$, this subcube is one of
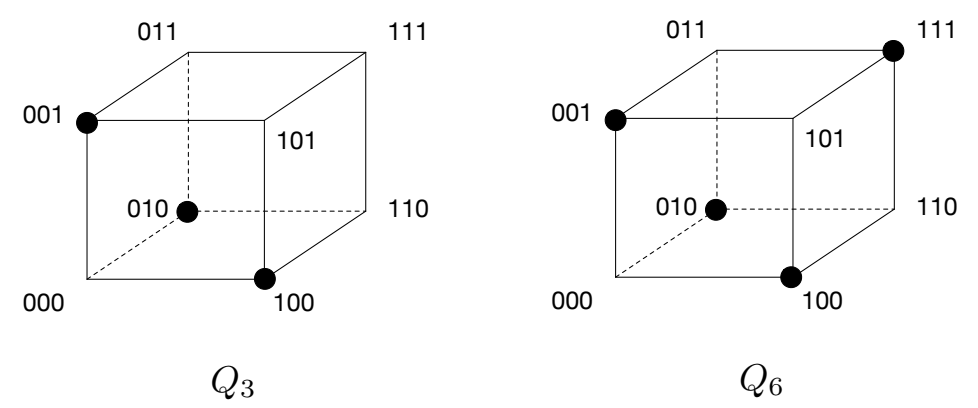

implying in turn that $\mathcal{C}$ has one of $Q_{3}, Q_{6}$ as a cuboid minor. This finishes the proof of Theorem 1.10 (i).

\subsection{Proof of Theorem 1.10 (ii)}

Take integers $n, k \geq 1$ and $S \subseteq\{0,1\}^{n}$ whose cuboid $\mathcal{C}$ has degree at most $k$ and does not have the packing property. We will show that $\mathcal{C}$ has a non-packing minor with at most $8 k+2$ elements, thereby proving Theorem 1.10 (ii). Recall that every cuboid minor of $\mathcal{C}$ also has degree at most $k$. We may therefore assume that every proper cuboid minor of $\mathcal{C}$ has the packing property. We may also assume that $\mathcal{C}$ has none of $\Delta_{3}, Q_{6}$ as a minor. If $\mathcal{C}$ does not pack, it then follows from Theorem 1.10 (i) that $\mathcal{C}$ itself has at most $8 k+2$ elements, so we are done. Otherwise, $\mathcal{C}$ packs. We will prove that every non-packing minor of $\mathcal{C}$ has at most $k$ elements.

Claim 1. C has two disjoint members.

Proof of Claim. Suppose otherwise. Since $\mathcal{C}$ packs, we have $\tau(\mathcal{C})=1$. We may therefore assume that every member of $\mathcal{C}$ contains element $2 n-1$ and excludes element $2 n$. Since $\mathcal{C}$ does not have the packing property, it has an mnp minor, which inevitably is also a minor of $\mathcal{C} /(2 n-1) \backslash 2 n$. This means that the proper cuboid minor $\mathcal{C} /(2 n-1) \backslash 2 n$ does not have the packing property, contradicting our minimal choice of $\mathcal{C}$.

Claim 2. Every proper deletion minor of $\mathcal{C}$ has the packing property, that is, every minor of $\mathcal{C}$ obtained after deleting at least one element has the packing property.

Proof of Claim. Consider the deletion minor $\mathcal{C} \backslash 2 n$. Since $2 n-1$ is a cover for this minor, it follows that every non-packing minor of $\mathcal{C} \backslash 2 n$ is actually a minor of $\mathcal{C} /(2 n-1) \backslash 2 n$, which as a cuboid minor of $\mathcal{C}$ has the packing property. Thus, $\mathcal{C} \backslash 2 n$ has the packing property. 
Claim 3. Every non-packing minor of $\mathcal{C}$ is obtained from $\mathcal{C}$ after contracting, for each $i \in[n]$, exactly one of $\{2 i-1,2 i\}$.

Proof of Claim. Let $\mathcal{C}^{\prime}$ be a non-packing minor of $\mathcal{C}$. By Claim 2, $\mathcal{C}^{\prime}$ is a contraction minor of $\mathcal{C}$. Take an index $i \in[n]$. Since the cuboid minor $\mathcal{C} /\{2 i-1,2 i\}$ has the packing property, $\mathcal{C}^{\prime}$ contains at least one of $2 i-1,2 i$. By Claim $1, \mathcal{C}$ has two disjoint members, which are still present in the contraction minor $\mathcal{C}^{\prime}$. Therefore, since $\mathcal{C}^{\prime}$ is non-packing, we see that $\tau\left(\mathcal{C}^{\prime}\right) \geq 3$, which in turn implies that $\mathcal{C}^{\prime}$ does not contain both $2 i-1,2 i$. Consequently, $\mathcal{C}^{\prime}$ contains exactly one of $2 i-1,2 i$, as claimed.

In particular, every non-packing minor of $\mathcal{C}$ is mnp. Let $\mathcal{C}^{\prime}$ be an mnp of $\mathcal{C}$. After a relabeling, if necessary, we may assume that $E\left(\mathcal{C}^{\prime}\right)=\{2 i-1: i \in[n]\}$, that is $S=\left\{\chi_{C^{\prime}}: C^{\prime} \in \mathcal{C}^{\prime}\right\}{ }^{6}$ Since every member of $\mathcal{C}^{\prime}$ has size at least two, the zero point 0 along with its neighboring unit points $e_{1}, \ldots, e_{n}$ are all in $\{0,1\}^{n}-S$. So the degree of $\mathbf{0}$ in $G_{n}\left[\{0,1\}^{n}-S\right]$ is $n$, implying in turn that $n \leq k$. In particular, $\left|E\left(\mathcal{C}^{\prime}\right)\right|=n \leq k$. This finishes the proof of Theorem 1.10 (ii).

\subsection{Proof of Theorem 1.10 (iii)}

Take integers $n, k \geq 1$, and a subset $S \subseteq\{0,1\}^{n}$ whose cuboid $\mathcal{C}$ is non-ideal and has degree at most $k$. Let $\mathcal{C}^{\prime}$ be an mni minor of $\mathcal{C}$. We will show that $\left|E\left(\mathcal{C}^{\prime}\right)\right| \leq k$, thereby finishing the proof of Theorem 1.10 (iii). We may assume that $\mathcal{C}$ is the only major of $\mathcal{C}^{\prime}$ that is a cuboid minor of $\mathcal{C}$.

Claim 1. $\mathcal{C}^{\prime}$ is a contraction minor of $\mathcal{C}$, that is, $\mathcal{C}^{\prime}$ is obtained from $\mathcal{C}$ after contracting some elements.

Proof of Claim. Suppose, for a contradiction, that $\mathcal{C}^{\prime}$ is a minor of $\mathcal{C} \backslash 2 n$. Since $2 n-1$ is a cover of $\mathcal{C} \backslash 2 n$, and $\mathcal{C}^{\prime}$ is mni, it follows that $\mathcal{C}^{\prime}$ is a minor of $\mathcal{C} /(2 n-1) \backslash 2 n$. Thus, $\mathcal{C}^{\prime}$ has a major $\mathcal{C} /(2 n-1) \backslash 2 n$ that is a proper cuboid minor of $\mathcal{C}$, contradicting our minimal choice of $\mathcal{C}$.

Claim 2. $\mathcal{C}^{\prime}$ is obtained from $\mathcal{C}$ after contracting, for each $i \in[n]$, exactly one of $\{2 i-1,2 i\}$.

Proof of Claim. Take an index $i \in[n]$. Since $\mathcal{C} /\{2 i-1,2 i\}$ is a cuboid minor of $\mathcal{C}$, it is not a major of $\mathcal{C}^{\prime}$, so $\mathcal{C}^{\prime}$ contains at least one of $2 i-1,2 i$. Suppose, for a contradiction, that $\mathcal{C}^{\prime}$ contains both $2 i-1,2 i$. In particular, $\tau\left(\mathcal{C}^{\prime}\right)=2$, so by Theorem 4.6, there is a member of $\mathcal{C}^{\prime}$ containing both $2 i-1,2 i$. This corresponds to a member of the cuboid $\mathcal{C}$ containing both $2 i-1,2 i$, a contradiction. Hence, $\mathcal{C}^{\prime}$ contains exactly one of $2 i-1,2 i$, as required.

After a relabeling, if necessary, we may assume that $E\left(\mathcal{C}^{\prime}\right)=\{2 i-1: i \in[n]\}$, that is $S=\left\{\chi_{C^{\prime}}: C^{\prime} \in \mathcal{C}^{\prime}\right\}$. Since every member of $\mathcal{C}^{\prime}$ has size at least two, the zero point $\mathbf{0}$ along with its neighboring unit points $e_{1}, \ldots, e_{n}$ are all in $\{0,1\}^{n}-S$. So the degree of 0 in $G_{n}\left[\{0,1\}^{n}-S\right]$ is $n$, implying in turn that $n \leq k$. In particular, $\left|E\left(\mathcal{C}^{\prime}\right)\right|=n \leq k$, thereby proving Theorem 1.10 (iii).

\footnotetext{
${ }^{6}$ Such a relabeling keeps the underlying graph $G_{n}[S]$ the same, up to isomorphism.
} 


\section{Conclusion}

To understand what makes a clutter non-packing, we studied mnp clutters, and as the non-ideal ones are wellunderstood, we focused on ideal mnp clutters. These clutters, however, are poorly understood to the extent where we are not even able to answer the following seemingly simple question:

Question 7.1. Can an ideal minimally non-packing clutter have a member of size two?

For ideal mnp clutters with covering number 2 , however, the answer is no, and in fact, there is even a certificate - let us elaborate. It is proved in [5] that an ideal mnp clutter $\mathcal{C}$ with $\tau(\mathcal{C})=2$ has the so-called $Q_{6}$ property, that $\mathcal{C}$ has members of the form

$$
\begin{array}{lll}
C_{1}=I_{1} \cup I_{3} \cup I_{5} & C_{3} & =I_{2} \cup I_{3} \cup I_{6} \\
C_{2}=I_{1} \cup I_{4} \cup I_{6} & C_{4} & =I_{2} \cup I_{4} \cup I_{5}
\end{array}
$$

for some partition of its ground set into nonempty parts $I_{1}, I_{2}, I_{3}, I_{4}, I_{5}, I_{6}$. Therefore, since every element of $\mathcal{C}$ appears in a cover of size 2, there are three pairwise disjoint covers of size 2 . In particular, every member of $\mathcal{C}$ has size at least three.

Because proving properties of ideal mnp clutters directly has been elusive so far, we reduced these clutters even further where, on top of the usual deletion and contraction operations, we applied the additional operation of identifying coexclusive elements. Identifications revealed chains of ideal mnp clutters (see Theorem 1.5 (i) and the examples in $\S 4.1$ ) and demonstrated the importance of mnp clutters with covering number 2 (Theorem 1.5 (ii)). Furthermore, we were able to provide a qualitative characterization of irreducible ideal mnp clutters with covering number 2 (Theorem 1.6), and this was done by the rather surprising emergence of marginal cuboids - objects that naturally give rise to ideal non-packing clutters with covering number 2 . We then presented an explicit class of marginal cuboids, and showed that the corresponding ideal non-packing clutters always have one of $Q_{6}, Q_{2,1}, Q_{10}$ as a minor (Theorem 1.9). We then extended Theorem 1.9 to bounded degree cuboids. We showed in Theorem 1.10 (ii) that, for each integer $k \geq 1$, there is a finite family of non-packing cuboids $\mathcal{N}_{k}$ such that, every non-packing cuboid of degree at most $k$ has a minor in $\mathcal{N}_{k}$. For instance, Theorem 1.9 shows that $\mathcal{N}_{2}=\left\{Q_{6}, Q_{2,1}, Q_{10}\right\}$.

In the vaguest terms, results such as Theorem 1.9 and Theorem 1.10 were within reach because most nonpacking cuboids have one of $\Delta_{3}, Q_{6}$ as a minor. Taking this perspective, a possible line of future research is to look for an explicit family $\mathcal{N}$ of non-packing clutters and a qualitative structural property $\mathcal{P}$ on cuboids such that the following holds:

If a non-packing cuboid has no minor in $\mathcal{N}$, then the cuboid satisfies $\mathcal{P}$.

A few late notes. Since the paper has been submitted, there has been a few updates; let us take this time to briefly discuss them. Take an integer $n \geq 1$ and a set $S \subseteq\{0,1\}^{n}$.

(1) We say that $S$ is cube-ideal if its convex hull is described using hypercube and generalized set covering inequalities. The careful reader will notice that we have shown that $S$ is cube-ideal if, and only if, the cuboid 
of $S$ is an ideal clutter. Cube-idealness has been studied in a follow-up paper [1]. For instance, it is shown that for a binary space, cube-idealness is the same as the rich and beautiful property of sums of circuits.

(2) We say that $S$ is polar if either all the points in $S$ agree on a coordinate or $S$ contains antipodal points. Notice that $S$ is polar if, and only if, its cuboid packs. Note that a marginal cuboid is the cuboid of a cube-ideal, non-polar set. We say that $S$ is strictly polar if every set obtained from $S$ after a series of 0 - and 1-restrictions is polar. Strict polarity has been further studied in [1]. For instance, it is shown that the following conjecture of Cornuéjols, Guenin and Margot [5],

(?) Every ideal minimally non-packing clutter has covering number 2. (?) which was briefly mentioned in this paper, is equivalent to the following conjecture:

(?) If $S$ is cube-ideal and strictly polar, then the cuboid of $S$ has the packing property. (?)

(3) Using Mantel's Theorem on maximal triangle-free graphs, the constants $4 k+2,8 k+2$ in Theorem 1.10 have been improved to $2 k+2,4 k+2$, respectively [1].

(4) A clutter is delta free if it has no delta minor. We showed in Theorem 2.3 that testing delta-free-ness can be done efficiently. In a follow-up paper, geometric aspects of delta free clutters and delta minors have been studied [3].

\section{Acknowledgements}

We are grateful for the support of the Hausdorff Institute for Mathematics in Bonn, Germany, as well as the organizers of its trimester program on Combinatorial Optimization; part of this work was carried out during our stay there. This work was also supported in part by NSF grant CMMI1560828 and ONR grant N00014-1210032, as well as an NSERC CGS D3 grant. We thank Michele Conforti for helpful discussions. We would also like to thank two referees who carefully read the paper; their suggestions improved the presentation of the paper.

\section{References}

[1] Abdi, A., Cornuéjols, G., Guričanová, N., Lee, D.: Cuboids, a class of clutters. Submitted.

[2] Abdi, A., Fukasawa, R., Sanità, L.: Opposite elements in clutters. To appear in Math. Oper. Res.

[3] Abdi, A. and Pashkovich, K.: Delta minors, delta free clutters, and entanglement. To appear in SIAM J. Discrete Math.

[4] Conforti, M., Cornuéjols, G., Zambelli, G.: Integer programming. Springer (2014)

[5] Cornuéjols, G., Guenin, B., Margot, F.: The packing property. Math. Program. Ser. A 89, 113-126 (2000) 
[6] Cornuéjols, G. and Lee, D.: On some polytopes contained in the 0,1 hypercube that have a small Chvátal rank. IPCO 2016, Louveaux, Q., Skutella, M. eds, LNCS 9682, 300-311 (2016)

[7] Edmonds, J. and Fulkerson, D.R.: Bottleneck extrema. J. Combin. Theory Ser. B 8, 299-306 (1970)

[8] Fulkerson, D.R.: Blocking and anti-blocking pairs of polyhedra. Math. Program. 1, 168-194 (1971)

[9] Guenin B.: Perfect and ideal 0, \pm 1 matrices. Math. Oper. Res. 23(2), 322-338 (1998)

[10] Isbell, J.R.: A class of simple games. Duke Math. J. 25(3), 423-439 (1958)

[11] Lehman, A.: On the width-length inequality. Math. Program. 17(1), 403-417 (1979)

[12] Lehman, A.: The width-length inequality and degenerate projective planes. DIMACS Vol. 1, 101-105 (1990)

[13] Lovász, L.: Minimax theorems for hypergraphs. Lecture Notes in Mathematics 411, Springer-Verlag 111$126(1972)$

[14] Lucchesi, C.L. and Younger D.H.: A minimax relation for directed graphs. J. London Math. Society 2(17), 369-374 (1978)

[15] Nobili P. and Sassano A.: (0, \pm 1$)$ ideal matrices. Math. Program. 80(3), 265-281 (1998)

[16] Schrijver, A.: A counterexample to a conjecture of Edmonds and Giles. Discrete Math. 32, 213-214 (1980)

[17] Seymour, P.D.: On Lehman's width-length characterization. DIMACS Vol. 1, 107-117 (1990)

[18] Seymour, P.D.: The forbidden minors of binary matrices. J. London Math. Society 2(12), 356-360 (1976)

[19] Seymour, P.D.: The matroids with the max-flow min-cut property. J. Combin. Theory Ser. B 23, 189-222 (1977)

[20] Woodall, D.R.: Menger and Kőnig systems. Lecture Notes in Mathematics 624, Springer-Verlag 620-635 (1978) 